\title{
On mechanisms of impurity leakage and retention in the tokamak divertor
}

\author{
I.Yu.Senichenkov ${ }^{1}$, E.G.Kaveeva ${ }^{1}$, E.A.Sytova ${ }^{1,2,3,4,5}$, V.A.Rozhansky ${ }^{1}$, \\ S.P.Voskoboynikov ${ }^{1}$, I.Yu.Veselova ${ }^{1}$, D.P.Coster ${ }^{2}$, X.Bonnin ${ }^{5}$, F.Reimold ${ }^{6}$, \\ and the ASDEX-Upgrade Team. \\ ${ }^{1}$ Peter the Great St.Petersburg Polytechnic University, 195251, Polytechnicheskaya ul., 29, Saint \\ Petersburg, Russia \\ ${ }^{2}$ Max-Planck Institut für Plasmaphysik, EURATOM Association, D-85748 Garching, Germany \\ ${ }^{3}$ Ghent University, Ghent, Belgium \\ ${ }^{4}$ Aix-Marseille Université, Marseille, France \\ ${ }^{5}$ ITER Organization, 13067 St. Paul-lez-Durance, France \\ ${ }^{6}$ Max-Planck-Institut für Plasmaphysik, EURATOM Association, 17491 Wendelsteinstr. 1, \\ Greifswald, Germany
}

\begin{abstract}
Impurity seeding into a tokamak divertor for radiative cooling is considered as a tool for achieving detached/semi-detached regimes required to meet the condition of acceptable heat loads on divertor plates. Experiments aimed at searching of the operational window with a significant reduction of poloidal heat fluxes due to the impurity radiation and without the decreasing of confinement are performed on many tokamaks. Critical issue in these experiments is which fraction of impurities is retained in the divertor region and which is extracted upstream to the scrape-off layer (SOL). In the present paper a physical mechanism of impurity transport from a divertor towards upstream and back to the divertor is analyzed. It is demonstrated that the widespread concept that the impurity leaks if the parallel thermal force exceeds the friction due to main ions and retains otherwise - is not correct. In contrast, the impurity leaks if it crosses the stagnation point of impurity ion poloidal velocity profile before the ionization, and retains if it ionizes closer to the target than the location of that stagnation point. Thus the leakage efficiency depends on the relative spatial positions of the impurity atom ionization source and the stagnation point of the impurity ion poloidal velocity profile. The impurity ion poloidal velocity is a sum of poloidal projection of its parallel velocity and the $E \times B$ drift velocity, where the former should be defined from the parallel impurity force balance equation. It is demonstrated that the solution of this equation may be approximated by the balance of friction and thermal forces in all regimes, while other terms are smaller. This allows for expressing the impurity parallel velocity through the main ion one and makes the distribution of the parallel (poloidal) fluxes of the main ions, including Pfirsch-Schlüter (PS) fluxes and $E \times B$ drift fluxes, to be an important element of the impurity transport. It is shown that impurity distribution in the edge plasma is rather sensitive to the value of the impurity ion ionization potential. This analysis is supported by the simulation results obtained for the ASDEX Upgrade tokamak with various seeding rates of $\mathrm{N}$ and Ne with the SOLPS-ITER code. The importance of inclusion of self-consistent drift flows is demonstrated by the comparison to result of corresponding simulations with drifts turned off.
\end{abstract}

Keywords: plasma edge, SOL, impurity seeding, detachment, electric field, drift, transport codes, SOLPS-ITER

PACS numbers: $52.55 . \mathrm{Fa}, 52.65 . \mathrm{Kj}$

Submitted to: Plasma Phys. Control. Fusion 


\section{Introduction}

The tokamak divertor was originally designed to reduce the impurity content in the confined plasma compared to a limiter configuration, and now is considered as an essential element of modern tokamaks and future thermonuclear reactors. The general idea was to shift the neutral source due to the wall recycling further from the confined plasma, so that neutrals should ionize before reaching the separatrix. After ionization, most impurity ions should be dragged back to the targets due to friction with the main ions. However, it soon became evident that some fraction of impurity ions leaks from the divertor and may reach up to the equatorial midplane. The widely accepted explanation for the leakage is the account of a thermal force, which drags impurity ions upstream if it overcomes the friction by main ions [1]. In this picture it is usually assumed that the main ion velocity is directed towards the target everywhere from the midplane to the target [2], so that the friction drags impurities downstream. However, as it is demonstrated below, the situation is not so simple.

In recent years the understanding of the mechanism for impurity retention/leakage has become more pressing as the impurity seeding into the edge plasma is found to be a reliable way to reach the divertor detachment. This allows for reducing the target heat loads down to an acceptable level through impurity radiation, and attaining such a regime with impurity seeding and radiative divertor operation is considered essential for future thermonuclear devices [3, 4].

However, it is not always possible to find an operational window with high enough fraction of discharge power irradiated in the divertor region and without significant impurity penetration to the core, which may cause a reduction of confinement. It is demonstrated experimentally on ASDEX Upgrade [5, 6, 7], JET [8, 9], and Alcator C-mod [10] that such a window exists with nitrogen seeding, where the fraction of radiated power can reach up to $90 \%$, while the confinement reduction remains moderate, with an $H_{98}$ factor staying above 0.9 .

Experiments with other seeding gases (like Ne, Ar, Kr) [11, 12, 13], however, did not demonstrate such good features - either insufficient divertor radiation or a strong reduction of confinement is observed. The latter is usually explained by the noble gas ion penetration up to the midplane and further into the confined region, where it may lead either to the impurity inward drag by neoclassical pinch ( $\mathrm{Ne}$ and $\mathrm{W}$ ) or simply to strong radiation losses from the core $(\mathrm{Kr})$. In both cases the radiative losses from the core become too large so that plasma may transit back to L-mode or even go into radiative collapse.

Numerical modeling of seeded discharges [14, 15, 16, 17] leads to a similar conclusion: nitrogen seeding allows for operation with an acceptable level of core confinement and high radiative fraction in the divertor, while for noble gases to find such an operational window is more difficult. These result might indicate that noble gases leak from the divertor more efficiently than the nitrogen. However, the impurity transport mechanisms still remain unclear, and especially important is the question of what is the key difference between different seeded gases [18].

Practically, for thermonuclear devices operating with tritium, one may want to avoid nitrogen seeding since it may lead to formation of radioactive ammonia molecules which would require specific treatment. From this point of view, neon seeding is preferable on ITER, and searching for an operational regime with high divertor radiative losses and low Ne concentration in the main plasma is underway now. Since ITER is not yet built, this problem may be solved only through numerical modeling.

Over the years, ITER divertor modeling was done with the SOLPS package [19, 20], and has recently been updated to SOLPS-ITER [21, 22]. This package is based on the B2.5 plasma solver (version 5.2) [23] and the Eirene Monte-Carlo kinetic code [24] for neutrals. The plasma solver 
includes a full self-consistent model for $E \times B$ drift, $\nabla B$ drift, viscosity currents and electric potential, based on Braginsky [28] set of equations. Recently the expressions for ion-impurity friction and thermal force were revised based on kinetic calculations [25] in order to lift the trace impurity assumption and obtain a correct form of the impurity force balance at arbitrary concentrations. Eirene code models the neutral distribution function with account of neutral-plasma interaction, including ionization, charge-exchange processes, molecule dissociation etc., and produces the source terms for plasma continuity, momentum balance and energy balace equations.

In the present paper the SOLPS-ITER package is used to model nitrogen seeded discharges in the ASDEX Upgrade (AUG) tokamak in order to investigate the transition to detachment with increasing seeding rate. We do not attempt a detailed reproduction of the experimental observations, but rather focus on a detailed comparison to another modeling scenario where nitrogen is replaced with neon while all other parameters are kept unchanged. Such a comparison starts from very low seeding rates so that both impurity gases do not affect the main plasma, and we increase the seeding rate progressively to study the difference between nitrogen and neon transport during the transition to detachment.

It is demonstrated that the friction force balances the thermal force in almost all regimes and everywhere throughout the SOL for the impurity, and that the parallel velocity of impurity may be found from this balance. The inertia, pressure gradient and electric forces are found to be less significant. Since the friction force depends on main ion parallel velocity, the distribution of main ion parallel (and poloidal) fluxes, including Pfirsch-Schlüter (PS) fluxes and $E \times B$ drift fluxes, becomes one of the key elements which determines the impurity dynamics. In this sense the inclusion of drifts and currents is an essential ingredient to simulations aimed at a self-consistent understanding of the mechanisms responsible for impurity transport.

These mechanisms are found to be more complicated than a simple competition of the friction and thermal forces, as it is widely accepted [1]. In fact, the ratio of impurity flux directed upstream to the one directed towards the target is defined by the fraction of impurity neutrals, which are ionized further from the target than the location of impurity poloidal flow stagnation point. Then those impurities are dragged upstream by the friction of main ions. The poloial velocity of impurity differs from the poloidal projection of its parallel velocity mainly due to the contribution of $E \times B$-drift and to a lesser extent by $\nabla B$-drift velocity and diffusion, so we again return to the importance of inclusion of drifts and currents. It is demonstrated that the $E \times B$ and $\nabla B$ drifts play a crucial role in the main ion (and, consequently, impurity too) poloidal velocity profile [27] simply by a comparison to an additional modeling scenario where these drifts and currents are turned off.

It is shown that impurity distribution in the edge plasma is very sensitive to the value of impurity neutral ionization potential, and, as a consequence, to the ionization rate $\left\langle\sigma_{\text {ion }} v\right\rangle$.

In particular, the key difference between nitrogen and neon distribution consists in the fact that neon ionizes further upstream than nitrogen, where the temperature is higher. This results in a larger fraction of neutrals which are ionized above the location of the impurity ion poloidal flow stagnation point for neon than for nitrogen, and thus the fraction of leaking neon ions is larger.

Naturally, the quantitative result (the location of impurity poloidal flow stagnation point and the spatial distribution of ionization source) depends on the divertor working regime, and may be different at the inner and outer targets. However, the general prediction is that neon leaks more efficiently than nitrogen, because neon ionizes further from the target than nitrogen, and thus has more chances to appear after the ionization in the zone where its poloidal flow is already directed upstream.

This paper is organized as follows. In Section 2 the main mechanism of impurity 
leakage/retention is proposed and briefly discussed. In Section 3 all the statements made in Section 2 are supported by the modeling. The ASDEX Upgrade shot \#28903 is described, which data were used to prepare the modeling scenarios. Then these scenarios are described and the main results of the simulations are presented. Then the impurity transport is described in detail. The force balance is shown, and it is demonstrated how the mechanisms of leakage work for neon and nitrogen in the modelled scenarios considered. Importance of main ion poloidal flow pattern details are emphasized, and the role of Pfirsch-Schlüter fluxes is demonstrated. In the same Section it is shown how results change if drifts and currents are turned off, especially considering the poloidal fluxes of main ions and impurities. In Section 4 it is shown that impurity retention becomes more pronounced with increasing seeding rate and with transition to detachment at the outer target. To explain this effect it is necessary to consider the changes in the main ion flow pattern, especially the reduction of $E \times B$ drift flux in the Private Flux Region (PFR). The paper then ends with the Conclusions.

\section{Mechanisms of impurity leakage and/or retention}

Let us consider the qualitative picture of impurity flow pattern in the tokamak Scrape-Off Layer (SOL) and divertor region. We start from the simplest case when the plasma consists of main ions (deuterium is assumed) and only one trace impurity, which may be either nitrogen or neon. The small amount of impurity guarantees that the effect of the impurity on the tokamak regime is negligible. This means that pedestal profiles, target profiles, the main ion SOL flow pattern and other parameters are not affected by this trace impurity, and would be the same as for a pure deuterium plasma.

The simplest 1D models of impurity retention/leakage, such as those described in the book of Stangeby [1], are based on the assumption that the flow of the main ions to the targets is a step function - it is directed to the targets in some region near the targets and is zero further upstream. This assumption is however too restrictive: there are upstream flows of the main ions from the ionization region near the targets and there are Pfirsch-Schlüter flows which close the divergent part of the $\nabla B$ and $E \times B$ drifts.

Hence, let us consider the stationary impurity force balance in the SOL and its projection in the direction of the magnetic field.

$$
\begin{array}{r}
\left(\nabla \cdot\left(m_{\mathrm{imp}} n_{\mathrm{imp}} \overrightarrow{\mathbf{u}}_{\mathrm{imp}} \overrightarrow{\mathbf{u}}_{\mathrm{imp}}\right)\right)_{\|}=-\nabla_{\|}\left(n_{\mathrm{imp}} T_{i}\right)-Z_{\mathrm{imp}} e n_{\mathrm{imp}} \nabla_{\|} \varphi+m_{e} n_{e} n_{\mathrm{imp}}\left\langle\sigma_{e} v\right\rangle\left(u_{\| e}-u_{\| \mathrm{imp}}\right)+ \\
m_{D} n_{D} n_{\mathrm{imp}}\left\langle\sigma_{i} v\right\rangle\left(u_{\| D}-u_{\| \mathrm{imp}}\right)+\alpha_{\mathrm{imp} i} Z_{\mathrm{imp}}^{2} n_{\mathrm{imp}} \nabla_{\|} T_{i}+\alpha_{\mathrm{imp}} Z_{\mathrm{imp}}^{2} n_{\mathrm{imp}} \nabla_{\|} T_{e}+S_{\| \text {ion }}^{\text {mom }}
\end{array}
$$

Here $n_{D}$ is the main ion (deuterium) density, $m_{D}=2 m_{p}$ is the deuterium ion mass $\left(m_{p}\right.$ is the proton mass), $m_{\mathrm{imp}}, Z_{\mathrm{imp}}, n_{\mathrm{imp}}, \overrightarrow{\mathbf{u}}_{\mathrm{imp}}$ are impurity ion mass, impurity electric charge, impurity density and impurity velocity, $m_{e}, n_{e}$ and $T_{e}$ are the electron mass, density and temperature, $T_{i}$ is the ion temperature, which is assumed to be common for main ions and impurities, $u_{\| e}=\left(\overrightarrow{\mathbf{u}}_{\mathrm{e}} \cdot \overrightarrow{\mathbf{B}} / B\right)$, $u_{\| D}=\left(\overrightarrow{\mathbf{u}}_{\mathrm{D}} \cdot \overrightarrow{\mathbf{B}} / B\right), u_{\| \text {imp }}=\left(\overrightarrow{\mathbf{u}}_{\mathrm{imp}} \cdot \overrightarrow{\mathbf{B}} / B\right)$ are projections of electrons, main ions and impurity velocities to the direction of the magnetic field, the index "imp" stands for the impurity ion (more accurately - for any ionization state of the impurity ions, excluding the neutral state). The left hand side of Eq. (1) is the inertia term, in the right hand side stand impurity pressure gradient, electric force, impurity friction by electrons, impurity friction by main ions, thermal forces due to friction by main ions and electrons and parallel momentum source due to impurity 
ionization/recombination/charge exchange. In expressions for friction forces $\sigma_{i}$ is a (transport) crosssection for momentum exchange between impurity and deuterium ions, so that $\left(n_{D}\left\langle\sigma_{i} v\right\rangle\right)^{-1}$ gives the distribution function averaged momentum exchange time for impurity ions, similarly the $\left(n_{e}\left\langle\sigma_{e} v\right\rangle\right)^{-1}$ is the momentum exchange time with regard to impurity-electron collisions, $\alpha_{\mathrm{imp}_{i, e}}$ are the kinetic coefficients of the order of unity which depend on the plasma composition, sort of impurity, its ionization state with charge $Z_{\text {imp }}$.

Due to relatively low SOL and divertor plasma temperatures and high collisionality one may expect that the largest force acting on impurities is the friction by main ion (see also [1]). Thus impurities are coupled to main ions by the strong friction in zeroth order crude approximation, and their velocities almost coincide. In the next approximation the impurity velocity adjusts to reduce the friction by main ions down to such a level so that it may be compensated by other terms in the force balance equation. To find the difference between main ion and impurity velocities one should find the largest term among others which would compensate the strong friction. These terms are naturally the thermal forces by main ions and electrons, which may be estimated as $\alpha_{\mathrm{imp}} Z_{\mathrm{imp}}^{2} n_{\mathrm{imp}} \nabla_{\|} T_{i}+\alpha_{\mathrm{imp} e} Z_{\mathrm{imp}}^{2} n_{\mathrm{imp}} \nabla_{\|} T_{e}$. Impurity pressure gradient $\nabla_{\|}\left(n_{\mathrm{imp}} T_{i}\right)$ is smaller due to a factor $Z_{\mathrm{imp}}^{2}$, the electric force $-Z_{\mathrm{imp}} e n_{\mathrm{imp}} \nabla_{\|} \varphi$ is of the order of $0.71 Z_{\mathrm{imp}} n_{\mathrm{imp}} \nabla_{\|} T_{e}$ and is also smaller — the ratio to the electron thermal force is about $0.71 /\left(\alpha_{\mathrm{imp}_{e}} Z_{\mathrm{imp}}\right)<1$. The inertia term (acceleration) is smaller than the pressure gradient if $u_{\mathrm{imp}}^{2}<T_{i} / m_{\mathrm{imp}}$. This criterion may be more severe for heavy impurity than for main ions, but there exist additional factor $Z_{\mathrm{imp}}^{2}$ between pressure gradient and thermal force. Thus one may expect that inertia is also small. Friction by electrons is expected to be smaller than friction by main ions by a factor of the order $\sqrt{m_{e} / m_{D}}$.

Strictly speaking, strong pressure gradients as well as momentum sources and sinks may exist in the momentum balance for any particular ionization states due to rapid spatial variation of its densiy, but they disappear after the summing of equations (1) over ionization states within one isonuclear sequence. Neglecting inertia term, this gives

$$
\begin{aligned}
0 \approx-\sum_{a} \nabla_{\|} & \left(n_{a} T_{i}\right)-\sum_{a} Z_{a} e n_{a} \nabla_{\|} \varphi+m_{e} n_{e} \sum_{a} n_{a}\left\langle\sigma_{e} v\right\rangle\left(u_{\| e}-u_{\| a}\right)+ \\
& m_{D} n_{D} \sum_{a} n_{a}\left\langle\sigma_{i} v\right\rangle\left(u_{\| D}-u_{\| a}\right)+\sum_{a} \alpha_{a i} Z_{a}^{2} n_{a} \nabla_{\|} T_{i}+\sum_{a} \alpha_{a e} Z_{a}^{2} n_{a} \nabla_{\|} T_{e}+S_{\| \text {neut }}^{\text {mom }} .
\end{aligned}
$$

Here the index " $a$ " stands for impurity ions within one isonuclear sequence (neutrals are excluded), $S_{\| \text {neut }}^{\text {mom }}$ gives the momentum source due to collisions with neutrals, and the presence of only one sort of impurity is assumed for simplicity. So, to investigate the net effect of impurity drag/leakage/retention the averaged (over ionization states) velocities and forces are of interest. Keeping in mind that $\left\langle\sigma_{i} v\right\rangle$ is proportional to the $Z_{\mathrm{imp}}^{2},\left\langle\sigma_{i} v\right\rangle=K Z_{\mathrm{imp}}^{2}$ this average velocity should be introduced as

$$
\left\langle u_{\| \operatorname{imp}}\right\rangle=\sum_{a} Z_{a}^{2} n_{a} u_{\| a} /\left\langle n_{\mathrm{imp}}\right\rangle
$$

with $\left\langle n_{\mathrm{imp}}\right\rangle=\sum_{a} n_{a} Z_{a}^{2}$ (which differs from $n_{\mathrm{imp}}$ total $=\sum_{a} n_{a}$ ). This velocity satisfies the equation

$$
m_{D} n_{D} K\left(u_{\| D}-\left\langle u_{\| \mathrm{imp}}\right\rangle\right)+\sum_{a} \frac{\alpha_{a i} Z_{a}^{2} n_{a}}{\left\langle n_{\mathrm{imp}}\right\rangle} \nabla_{\|} T_{i}+\sum_{a} \frac{\alpha_{a e} Z_{a}^{2} n_{a}}{\left\langle n_{\mathrm{imp}}\right\rangle} \nabla_{\|} T_{e} \approx 0,
$$

where the friction by electrons, pressure gradient, electric force (all three are summed over impurity ionization states) are neglected, as well as momentum source due to collisions with neutrals.

Finally, if the friction force is indeed larger than other forces which are neglected in the Eq. (4), one may expect that for each impurity ionization state its parallel velocity $u_{\| \text {imp }}$ almost coincides 
with the average velocity $\left\langle u_{\| \text {imp }}\right\rangle$. If it is the case, then the average velocity may be replaced by individual velocity of each ionization state in the Eq. (4), which gives for any ionization state

$$
m_{D} n_{D} n_{\mathrm{imp}}\left\langle\sigma_{i} v\right\rangle\left(u_{\| D}-u_{\| \mathrm{imp}}\right)+\alpha_{\mathrm{imp} i} Z_{\mathrm{imp}}^{2} n_{\mathrm{imp}} \nabla_{\|} T_{i}+\alpha_{\mathrm{imp} e} Z_{\mathrm{imp}}^{2} n_{\mathrm{imp}} \nabla_{\|} T_{e} \approx 0 .
$$

Here it is assumed implicitly that kinetic coefficients $\alpha_{\mathrm{imp}_{i}}$ are $\alpha_{\mathrm{imp}_{e}}$ are same for all ionization states within one isonuclear sequence, in accordance with [25].

If the approximation (5) is valid, it defines the local value of the parallel impurity velocity, assuming that the density $n_{D}$ and the parallel velocity $u_{\| D}$ of main ions together with the common temperature $T_{i}$ are known (and are not affected by the trace impurity). The validity of the Eq. (5) is supported by modeling results, see the next sections.

Now assume that the main ion parallel velocity has a stagnation point near the $\mathrm{X}$-point or even closer to the target, i.e. below this stagnation point the parallel velocity is directed towards the target and reaches the sound speed on its surface to satisfy the Bohm-Chodura criterion, and above this stagnation point the main ion parallel flow is directed upstream. For the discharges with $B \times \nabla B$ drifts directed towards the active X-point, this upstream flow, which was observed experimentally on many tokamaks [30, 31, 32, 33, 34, 35, 36, is to a large extent determined by the Pfirsch-Schlüter flow (in the near SOL) [37]. Another significant contribution to the upstream flows is so-called reversal flows, originating from the intensive ionization source in the near SOL and returning back to the target by radial diffusion and by downstream flows in the far SOL. The location of the stagnation point, naturally, may vary from one flux tube to another as one moves from the separatrix towards the far SOL due to different radial distributions of ionization sources and radial main ion transport.

The thermal force, which pulls impurities up towards regions of higher ion temperature, shifts the location of the stagnation point of the impurity parallel velocity towards the divertor with respect to the stagnation point of the main ion parallel velocity. Indeed, from the Eq. (5), assuming that the coordinate along the B-field line increases from the target towards upstream, one can see that thermal force(s) is (are) positive, so that the $\left(u_{\| D}-u_{\| \mathrm{imp}}\right)$ should be negative. Thus, the parallel velocity of main ions is negative (i.e. is directed downstream) at the location of impurity parallel flow stagnation point $\left(u_{\| \text {imp }}=0\right)$, while the parallel velocity of impurity is positive at the location of the main ion parallel flow stagnation point $\left(u_{\| D}=0\right)$. Since both parallel velocities are negative at the target, one concludes that the stagnation point of impurity parallel flow is located closer to the target than one of main ions. Further upstream the difference between impurity and main ion parallel velocities is again due to thermal force.

The poloidal flow pattern of the impurity can then be obtained by adding the poloidal $E \times B$ drift velocity and some other small terms, like the $\nabla B$ drift velocity, impurity diffusion, contributions from ion currents along the B-field, etc. Thus the locations of the stagnation points for poloidal and parallel impurity flows do not coincide. And it is worth mentioning once again here that drifts contribute to the value of impurity poloidal velocity two times. First, they affect the $u_{\| D}$, which the $u_{\| \text {imp }}$ depends on through the parallel projection of the force balance (5). Second, they define the difference between the poloidal projection of impurity parallel velocity $u_{\| \mathrm{imp}} \cdot B_{\text {pol }} / B$ and its poloidal velocity itself $u_{p o l}$ imp. As it will be demonstrated below from the modeling, the account of drifts and currents is essential.

The poloidal velocity is the definitive quantity for the ion leakage or retention. Naturally, those among the neutral atoms which ionize before reaching the stagnation point of the ion poloidal flow return back to the target. If the atom ionizes further upstream than the location of the stagnation point, that ion is dragged towards the midplane. And this is true both for main ions and for 


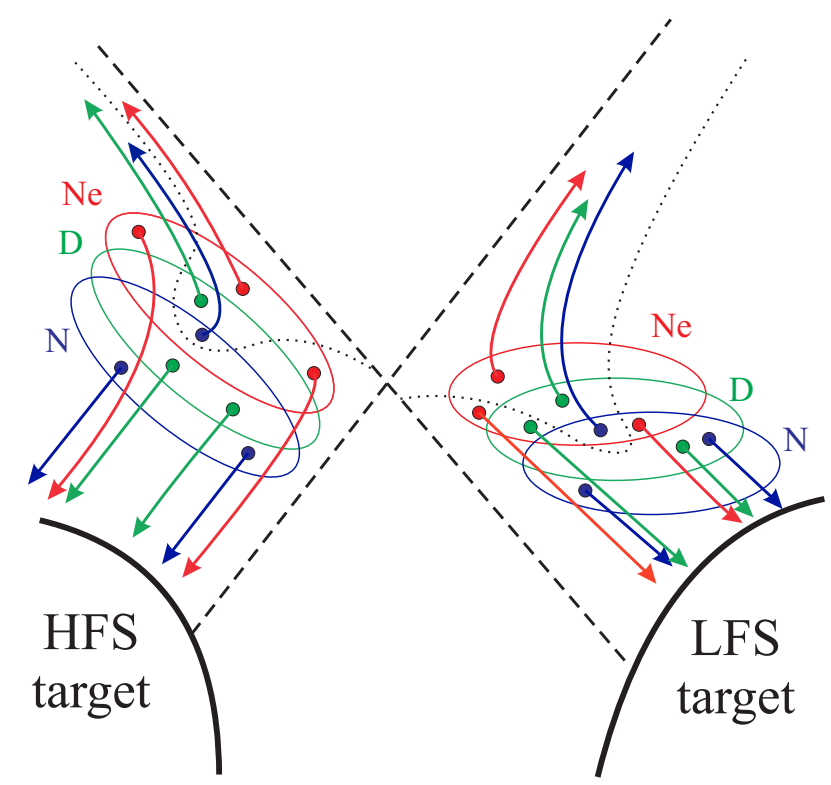

Figure 1. Zones of maximal ionization for deuterium (green ellipses), nitrogen (blue ellipses) and neon (red ellipses) near the X-point in the SOL. Dash lines schematically represent the stagnation points of poloidal flow (which is the same for all species if parallel velocities of all species coincide). Solid circles show points of ionization and arrows of corresponding color represent the poloidal flow directions.

impurities.

For the main ions, the majority of neutrals participate in the target recycling, i.e. return back to the target after ionization. This is only possible if these neutrals are ionized before reaching the stagnation point of the main ion poloidal flow. Those neutrals which can penetrate further upstream than this stagnation point before the ionization, after the ionization move towards the midplane. The leakage of such main ions/neutrals from the divertor is compensated by some kind of return flow, which may be the gas puff, the neutral flow from another divertor behind the vacuum vessel structures (if there are any), $\nabla B$-drift towards the Private Flux Region (PFR) and the drag towards the SOL from the PFR by $E \times B$ drift. In some situations this return flow is provided by simple radial diffusion and by reversal flow towards the divertor through the far SOL, since the ionization and leakage are more intensive in the near SOL than in the far SOL.

In other words, the main ion poloidal and parallel flow patterns form self-consistently with their density profile and profiles of both temperatures, which the ionization rate and the ionization source depend on. For trace impurities the situation is different in a sense that their parallel velocity and ionization source profiles - which depend on $n_{e}, T_{e}, T_{i}, u_{\| D}$ - are defined by main ions rather than by impurities themselves.

Now the different behavior of ions with different ionization potential, i.e. the effect of finite ionization potential (FIP), can be understood - the difference in behavior comes from the difference in ionization profiles. Let's assume for a moment that the thermal force in Eq. (5) is smaller than friction, so that parallel velocities of main ions and impurities almost coincide. Consequently, poloidal velocities almost coincide too, since they differ from parallel ones mainly by the $E \times B$ drift velocities. Since the electron temperature monotonically increases with the distance from the target, the location of maximal ionization will appear in the following order: nitrogen, deuterium, neon, see Figure 1. This statement requires additional comment: in spite of the fact that the ionization potential of nitrogen $(14.5 \mathrm{eV})$ is bigger than that of deuterium $(13.6 \mathrm{eV})$, the ionization cross-section $\sigma_{\text {ion }}$ and the ionization rate $\left\langle\sigma_{\text {ion }} v\right\rangle$ (angle brackets mean averaging over the Maxwellian distribution function) 
for nitrogen is about two times higher than that of deuterium for the temperature range of several eVs [38. The account of step ionization through deuterium molecular ionization, charge exchange with molecualr ions and other reactions, which is carefully done in the modeling (see sections below), does not change the relative positions of ionization peaks - the nitrogen starts to ionize at lower temperatures than deuterium, i.e. closer to the target. Since most of the deuterium neutrals are ionized in the zone where the poloidal flow of deuterium ions is directed downstream, the nitrogen neutrals after the ionization appear in the zone of nitrogen poloidal flow directed downstream too (mind that we forgot about the thermal force for a while!), i.e. the nitrogen tends to retain. For the neon the situation is the opposite: it ionizes further upstream than deuterium, i.e. in the zone where the deuterium ion poloidal flow is already directed upstream. Consequently, the poloidal velocity of neon should also be directed upstream in this zone, i.e. the neon leaks.

The account of thermal force increases the leakage efficiency, since it shifts the location of impurity poloidal flow stagnation point towards the target and thus makes it easier for impurity neutral to be ionized in the zone of upstream impurity poloidal flow. For the nitrogen this effect competes with the effect of higher ionization rate, while for the neon both effects lead to higher leakage efficiency than for deuterium. As a result, one would expect that the ratio of the number of leaking atoms to the number of retained ones is bigger for neon than for deuterium and for nitrogen, thus leading to higher nitrogen compression than neon one, $C_{\mathrm{N}}>C_{\mathrm{Ne}}$. For nitrogen and deuterium the value of $C_{\mathrm{N}} / C_{\mathrm{D}}$ (also known as the enrichment) is difficult to predict - it depends on the divertor working regime, the relative role of thermal force, the relative role of $\nabla B$-drift in the formation of poloidal deuterium velocity and so on, so this ratio may be computed only numerically.

This picture may explain roughly why it is relatively easy to obtain strong radiation losses from

the divertor without the loss of confinement with nitrogen seeding, while with neon seeding it may not be possible to find an operational point where strong radiation losses in the divertor can be obtained without some significant neon leakage towards the main plasma. However, it is worth mentioning again that the picture presented above is rather rough, and numerical calculations are required to obtain the exact value of neon compression for the regime of interest.

It is important to say now that the structure of main ion flow becomes very important for the impurity leakage and retention. In principle, it might be possible to control the location of main ion parallel flow stagnation point by varying the location of puff and pump (e.g. by fueling the discharge from the top of the machine and pumping near targets) and by inverting the toroidal magnetic field (thus reversing the Pfirsch-Schlüter flow) — like in the DIII-D experiment [13]. In those experiments the effective argon retention may be explained by the possible shift of the main ion poloidal flow stagnation point further upstream by effects mentioned above.

Generally speaking, for any particular tokamak with its power, geometry, upstream condition etc., the question whether any particular radiant is suitable for achieving the detached divertor without a notable damage to the core confinement, requires a separate investigation, including numerical modeling. In the present analysis we restrict ourselves by comparing nitrogen versus neon for ASDEX Upgrade conditions, leaving other devices and other radiants beyond the scope of this paper.

\section{Results of ASDEX Upgrade plasma edge modeling with trace impurities}

\subsection{Modeling results}

In this section we present the results of modeling by means of the SOLPS-ITER code, which gives the basis for the qualitative picture of impurity transport described above. 


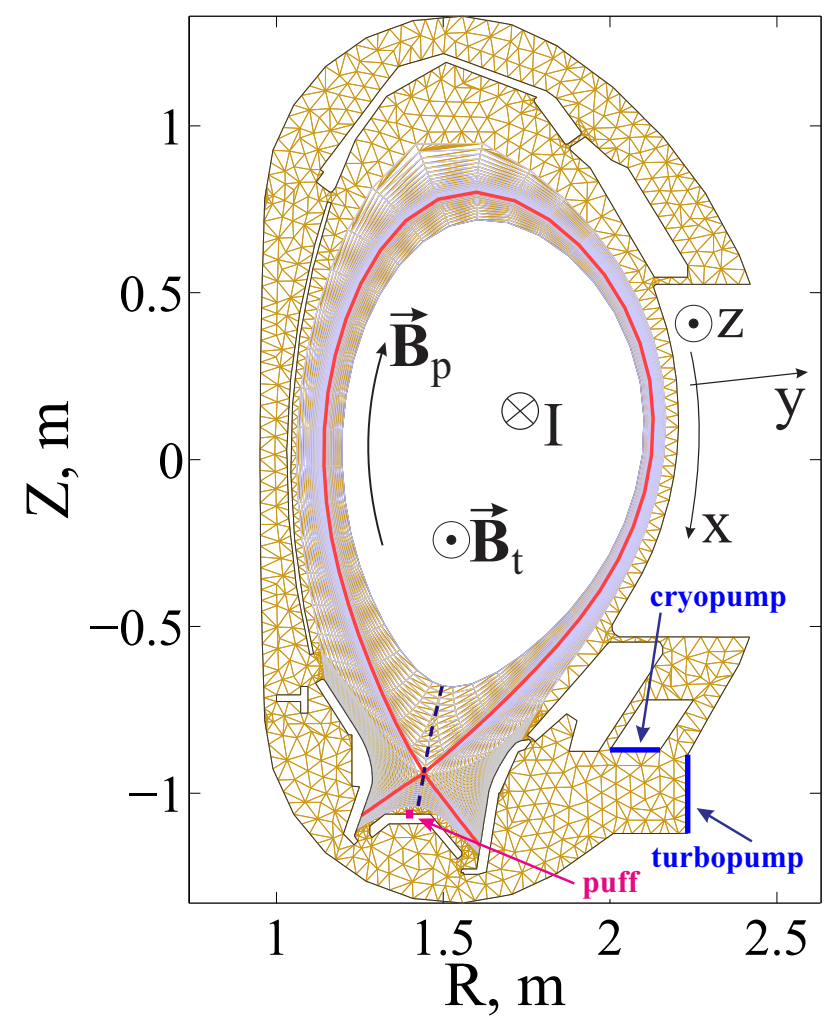

Figure 2. Computational meshes for B2.5 (violet quadrangular cells) and Eirene (dark-yellow triangular cells) used in the simulations. Shown are the in-vessel structures and locations of gas puff/seeding and pumping.

The modeling case is based on the ASDEX Upgrade shot \#28903 (see also [6, 17]), where an H-mode deuterium plasma was seeded with nitrogen. The auxiliary heating power (NBI + ICRH) in this discharge was $P_{\text {tot }}=8.2 \mathrm{MW}$, the toroidal magnetic field on axis was $B_{t}=2.5 \mathrm{~T}$, and the plasma current was $I_{p}=800 \mathrm{kA}$. The plasma fueling rate was $2 \cdot 10^{22} \mathrm{D}$ atoms $/ \mathrm{s}$, the seeding rate $3.6 \cdot 10^{21} \mathrm{~N}$ atoms $/ \mathrm{s}$ was applied after $t=2.8 \mathrm{~s}$, which led to the development of strong X-point radiation at about $t=3.6 \mathrm{~s}$ and to complete detachment of both targets after $t=5.5 \mathrm{~s}$.

The computational grid for the B2.5 and Eirene components of the SOLPS-ITER code suite was built from an experimentally measured equilibrium, and is shown in Figure 2.

The power crossing the core boundary of the computational domain is set to $P_{\text {core }}=5 \mathrm{MW}$ (thus taking into account the core radiation determined from bolometric tomography [17]), with the power ratio of electron to ion channel set to 3.2/1.8. The net deuterium flux through this boundary (representing the NBI particle source) is set to $8 \cdot 10^{20} \mathrm{~s}^{-1}$, the net impurity flux is set to zero. The poloidally averaged parallel velocity of deuterium ions at the core boundary is chosen to be $-50 \mathrm{~km} / \mathrm{s}$, representing the torque from the NBI. For the impurity parallel velocity the boundary condition of zero radial (across flux surfaces) gradient is applied.

On the wall side of the B2.5 computational domain, a leakage boundary condition is applied for all ion densities and both ion and electron temperatures, which means that the local radial particle or heat flux density is a small fraction $\left(10^{-2}\right.$ for all densities, $2 \cdot 10^{-2}$ for $T_{i}$ and $10^{-4}$ for $\left.T_{e}\right)$ of the convective sound speed flux for ions and electrons respectively. The radial gradients for all parallel velocities are set to zero.

Typical sheath boundary conditions for ion density, both electron and ion temperatures, ion poloidal velocities, current density and electric potential are applied on targets (see e.g. [23] for a full description). 


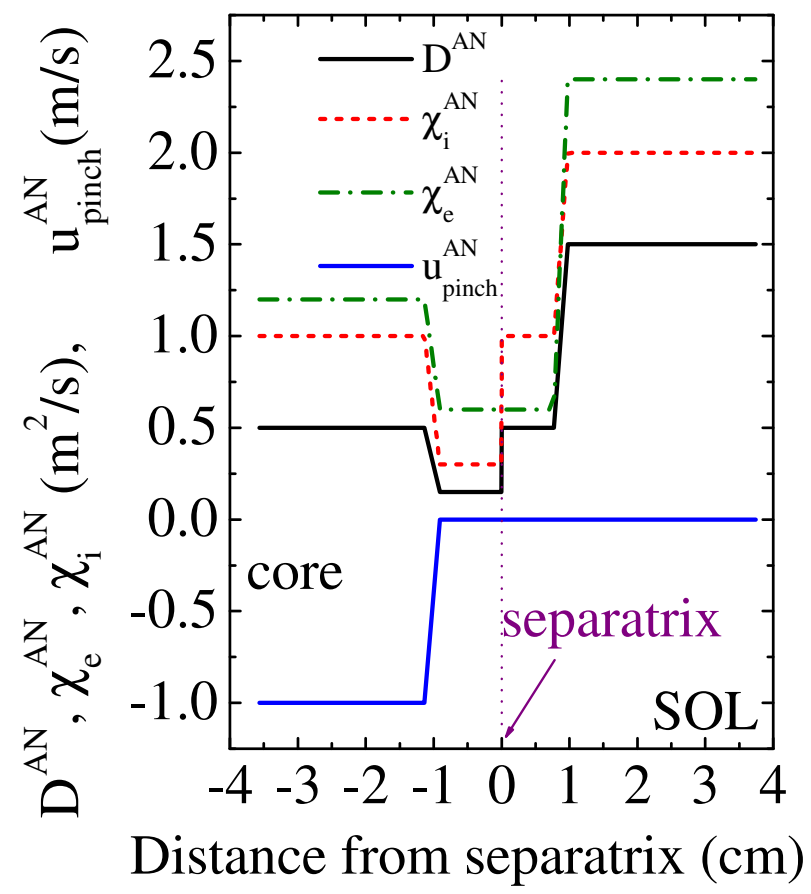

Figure 3. Anomalous transport coefficients used in the simulation plotted in equatorial midplane, Low Field Side (LFS). Positive abscissa values correspond to SOL, negative - to the confined region (core). $u_{\text {pinch }}^{\mathrm{AN}}$ is the anomalous pinch velocity, so that radial anomalous particle flux density is assumed to be $\Gamma_{y}=-D^{\mathrm{AN}}(\nabla n)_{y}+u_{\text {pinch }}^{\mathrm{AN}} n$.

The deuterium fueling rate is set to $2 \cdot 10^{22}$ atoms/s, as in the experiment, while the impurity seeding rate is varied from $2 \cdot 10^{17}$ atoms/s to $2 \cdot 10^{20}$ atoms/s for nitrogen and from $2 \cdot 10^{17}$ atoms $/ \mathrm{s}$ to $5 \cdot 10^{19}$ atoms/s for neon. Cases with higher seeding rates rapidly run to a radiative collapse due to enormous impurity penetration into the confined region through the cold spot above the X-point. Analysis of such scenarios is beyond the scope of this paper, it is a task for future investigations. In this section only the cases with seeding rates $2 \cdot 10^{18}$ atoms/s and below are considered in details, since for higer seeding rates it is no more possible to neglect the effects of impurities on plasma parameters.

Special notes should be given on the choice of anomalous transport coefficients. Since the SOLPS-ITER package does not allow at the moment for a self-consistent theory-based calculation of these coefficients, they need to be set explicitly. In the simulations presented here they were fitted so that the computed plasma profiles for the lowest impurity seeding rate at the equatorial midplane and at the targets match as best as possible the experimentally measured ones at $t=2.4$ s, i.e. just before the impurity seeding was applied. The transport coefficients obtained from this fitting process are plotted in Figure 3. Note that they all (including the pinch velocity) are the same for all ion species, i.e. both main ions and impurity ions. This last choice is made under the assumption that the anomalous transport is due to $E \times B$ drift in the fluctuating E-field of microturbulence, and, since $E \times B$ drift velocity is common for all species, anomalous transport coefficients are therefore common too.

Note that in spite of that the anomalous transport coefficients are poloidally constant, radial flux densities are poloidally dependent due to geometry effects and they have maximum at the outer midplane, where the distance between neighboring flux surfaces is minimal. The inclusion of poloidal dependence of the anomalous transport coefficients would increase the effect, and in some cases might improve the quantitative agreement between calculated and measured poloidal profiles, 
as in [42]. However, one should keep in mind that the problem of the turbulent transport coefficients calculations from the first principles for tokamak plasma in the vicinity of separatrix and in the SOL is far from its solution, so that the strength of such poloidal variation would be just another free input parameter for 2D fluid transport codes like SOLPS-ITER or EDGE2D. And also there exist simulation examples where the acceptable agreement between computed and experimental profiles is reached without (with small) the poloidal modulation [23, 41, 43].

The changes between profiles computed with and without the poloidal modulation of transport coefficients are expected to appear above the X-point, where the difference in the divergent part of radial turbulent fluxes would exist. However, as it is mentioned in [44], the distribution of particle ionization source is also important for the poloidal profile of parallel velocity, especially in the highrecycling regime modelled in the present paper. For this regime the parallel velocity near and below $\mathrm{X}$-points is defined to large extent by the ionization source profile rather than by poloidal distribution of the upstream flows, so no significant effect of upstream flow details on parallel velocities in divertor is expected.

The main plasma profiles along the equatorial midplane at the Low Field Side (LFS) are plotted in Figure 4 for different radiators and for different seeding rates in the range from $2 \cdot 10^{17}$ to $5 \cdot 10^{19}$ impurity atoms per second. One can see that for seeding rates $2 \cdot 10^{18}$ atoms $/ \mathrm{s}$ and below the main plasma parameters are identical independently of the seed gases - thus one can conclude that such low seeding rates keep the plasma in the trace impurity regime. In that seeding rate range, the target profiles are also identical, see Figures 5 and 6. Thus one can study the difference in behavior of different radiators for identical main plasma background. For seeding rates bigger than $2 \cdot 10^{18}$ atoms/s the trace impurity approximation fails, and one can see changes in main plasma parameters, mainly at outer target.

From Figures 5 and 6, one can see that even with the smallest amount of impurity the inner target is already detached, while the outer one is attached (operates in the high recycling regime). 2D plots of electron density and temperature and of total main ion source due to ionization (which includes direct electron impact ionization $n_{\mathrm{D}^{0}} n_{e}\left\langle\sigma_{\mathrm{ion}} v\right\rangle$, ionization from excited states, dissociation of molecular ions and other relevant processes) are plotted in Figures 7 and 8 for case with nitrogen seeding rate $2 \cdot 10^{18}$ at/s. Here one can see a formation of the High Field Side High Density (HFSHD) feature - the region of high electron density and low temperature near the inner target, which is observed in experiments.

With increasing seeding rate the outer target starts to detach too, and the transition to detachment may be reached with lower neon seeding rates than with nitrogen ones. Indeed, the target profiles, such as electron density and temperature, as well as the fraction of the irradiated power, are very similar to each other for the nitrogen seeded plasma with the seeding rate equal to $5 \cdot 10^{19} \mathrm{~s}^{-1}$ and the neon seeded plasma with the seeding rate $2 \cdot 10^{19} \mathrm{~s}^{-1}$, thus illustrating that different seeding rates of nitrogen and neon are required to reach the same degree of detachment. Another pair of modeling scenarios resulting in the same plasma profiles with different radiators corresponds to the nitrogen seeding rate $1.2 \cdot 10^{20} \mathrm{~s}^{-1}$ and neon seeding rate $5 \cdot 10^{19} \mathrm{~s}^{-1}$, demonstrating the same trend. Simultaneously, for the same seeding rate of different radiators the outer target is colder and denser for neon seeded plasma than for the nitrogen seeded one.

The calculated impurity density is shown in Figure 9. One can see that in spite of almost identical main plasma background (at least for seeding rates below $2 \cdot 10^{18} \mathrm{~s}^{-1}$ ) the neon and nitrogen distributions are significantly different even for the same seeding rate. Nitrogen tends to retain near the inner target, forming, like for deuterium, a strong density peak in the zone of low temperature. Neon, in contrast, does not form such a strong density peak but tends to leak from the inner target 

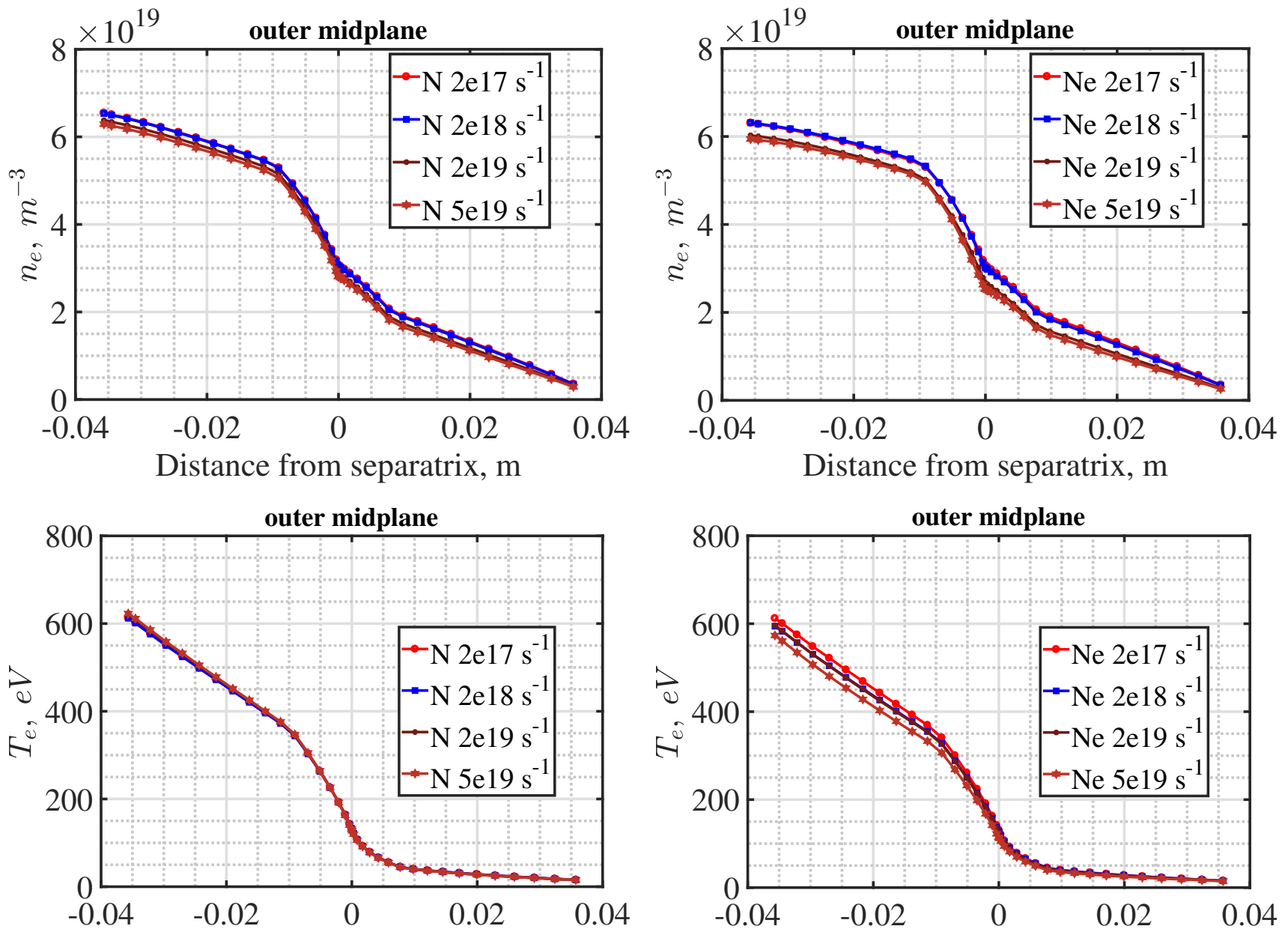

Distance from separatrix, $\mathrm{m}$

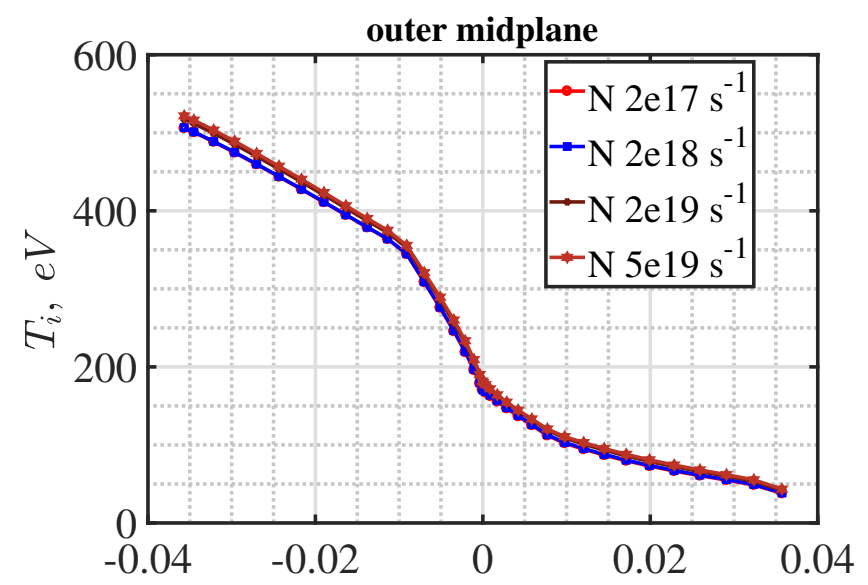

Distance from separatrix, $\mathrm{m}$

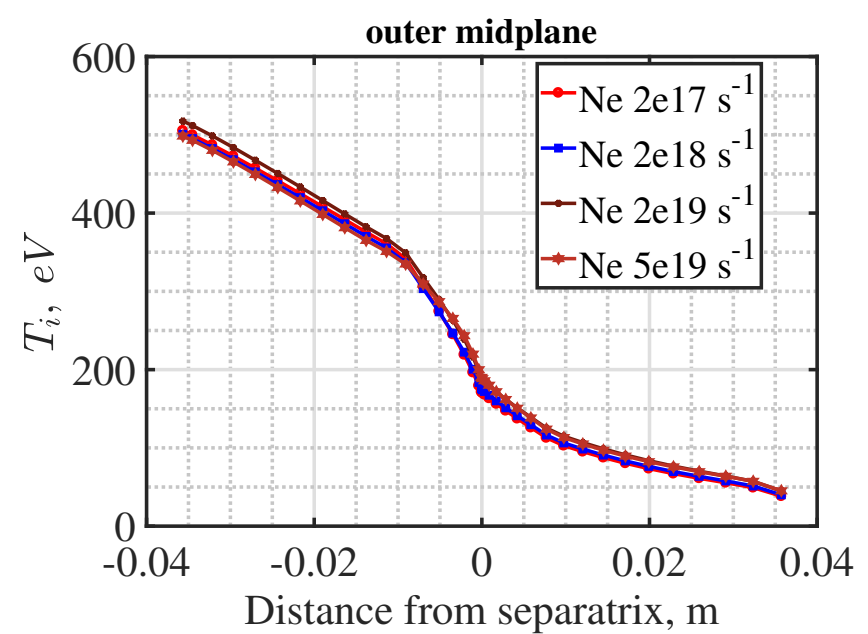

Figure 4. Main plasma profiles at the equatorial midplane, LFS, for different nitrogen and neon seeding rates. Like in Fig 4 positive abscissa values correspond to SOL, negative - to the confined region (core). 

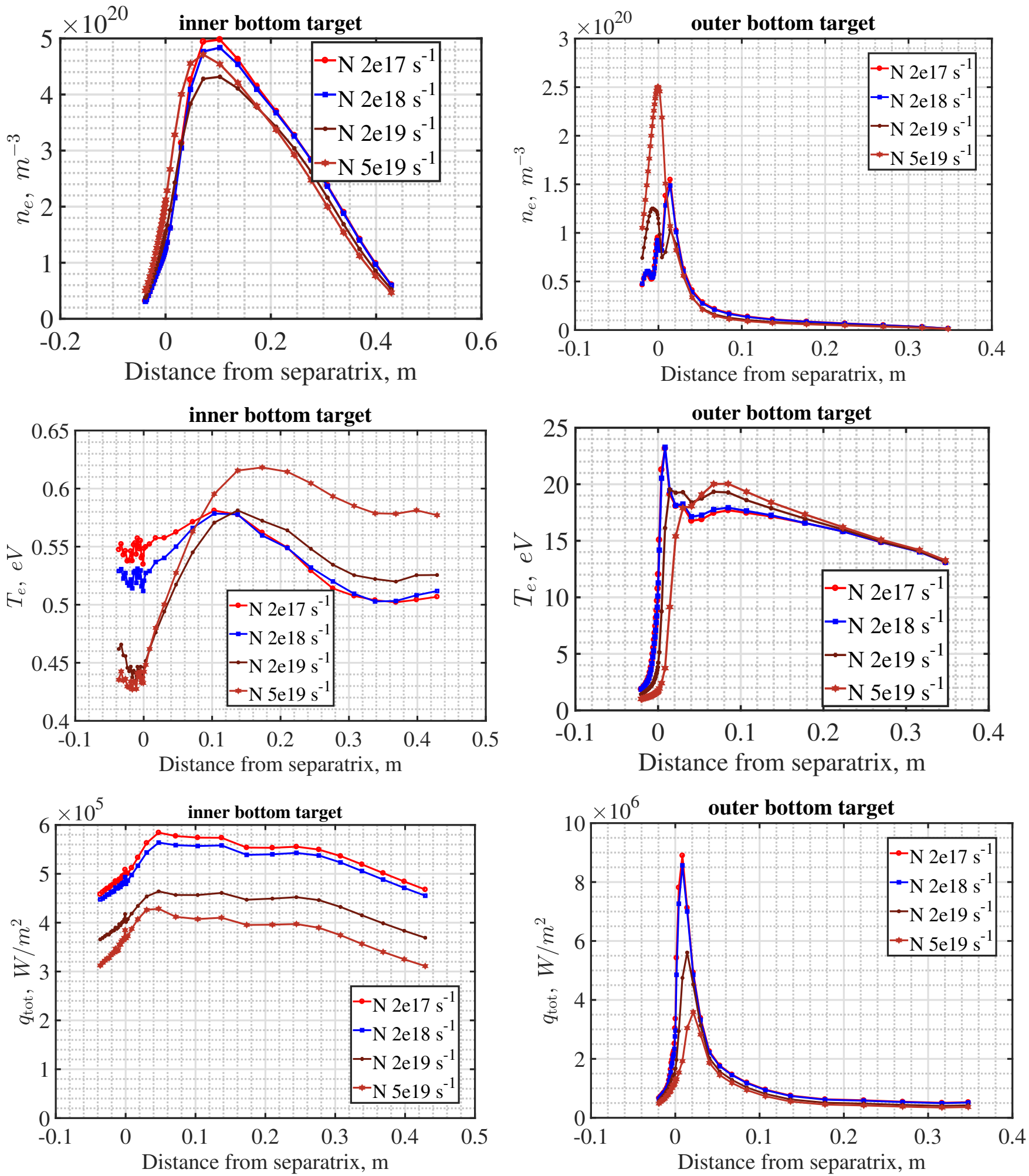

Figure 5. Electron density, electron temperature and heat flux density along targets for different nitrogen seeding rates. Positive abscissa values correspond to SOL, negative - to the PFR. 

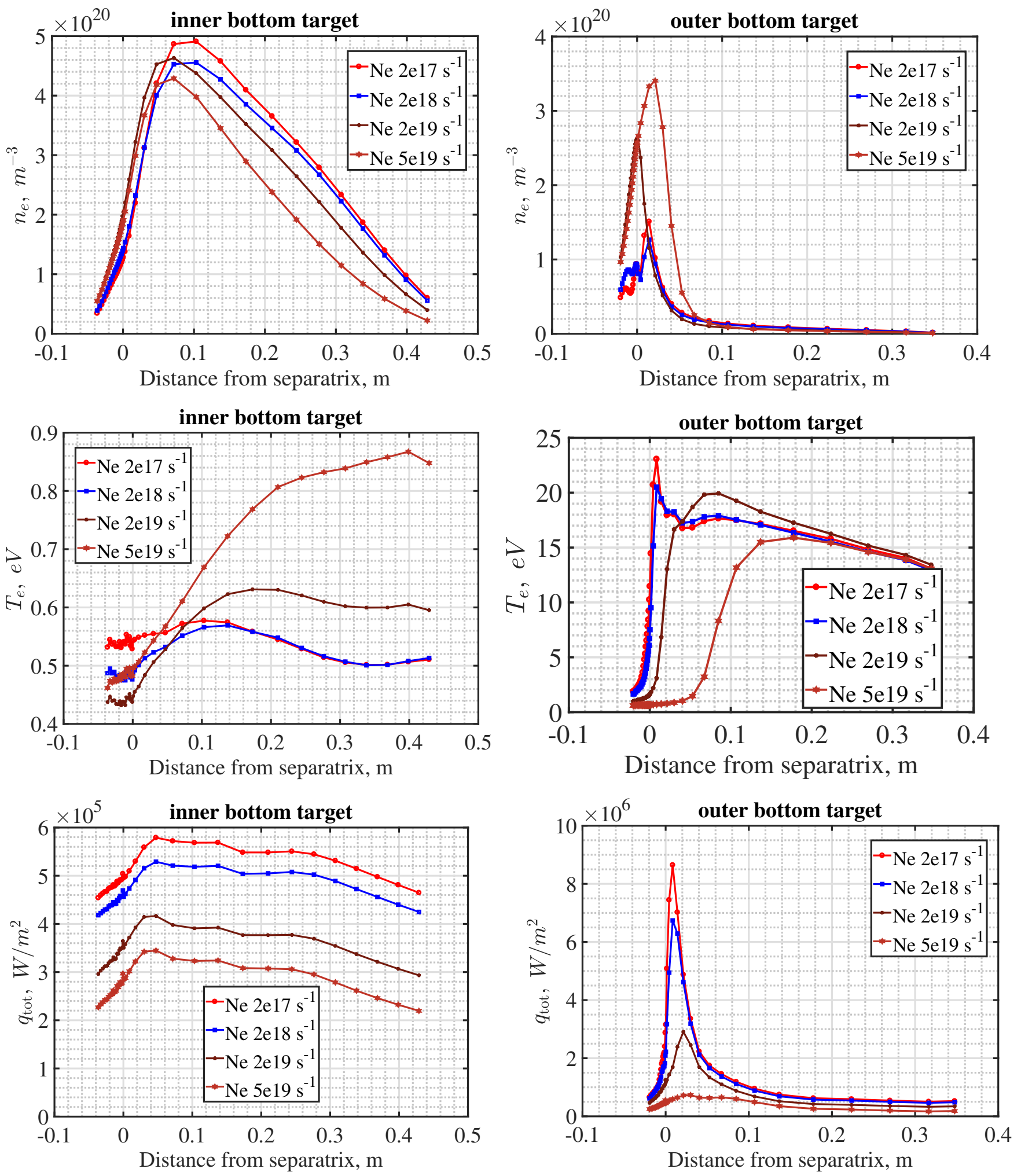

Figure 6. Electron density, electron temperature and heat flux density at targets for different neon seeding rates. Positive abscissa values correspond to SOL, negative - to the PFR. 

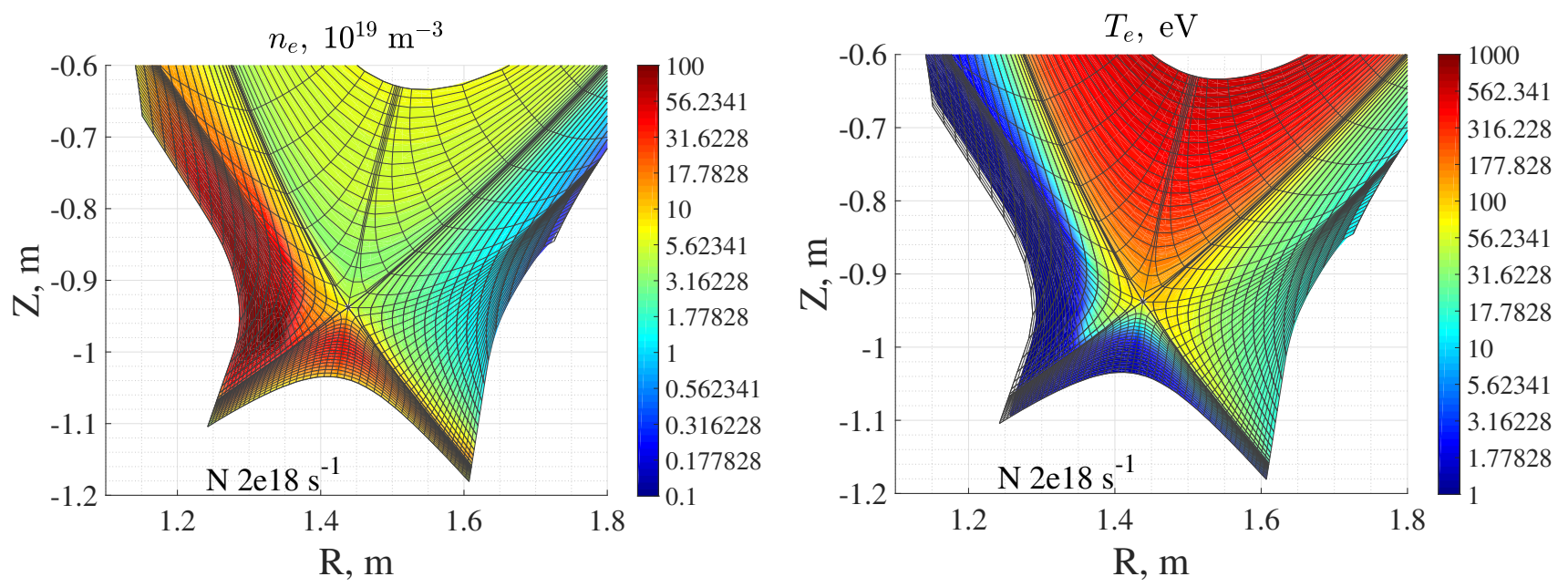

Figure 7. 2D contour plots of electron density and temperature in the vicinity of X-point and targets. Here and in other 2D figures white spots correspond to values below the minimum value on colorbar.

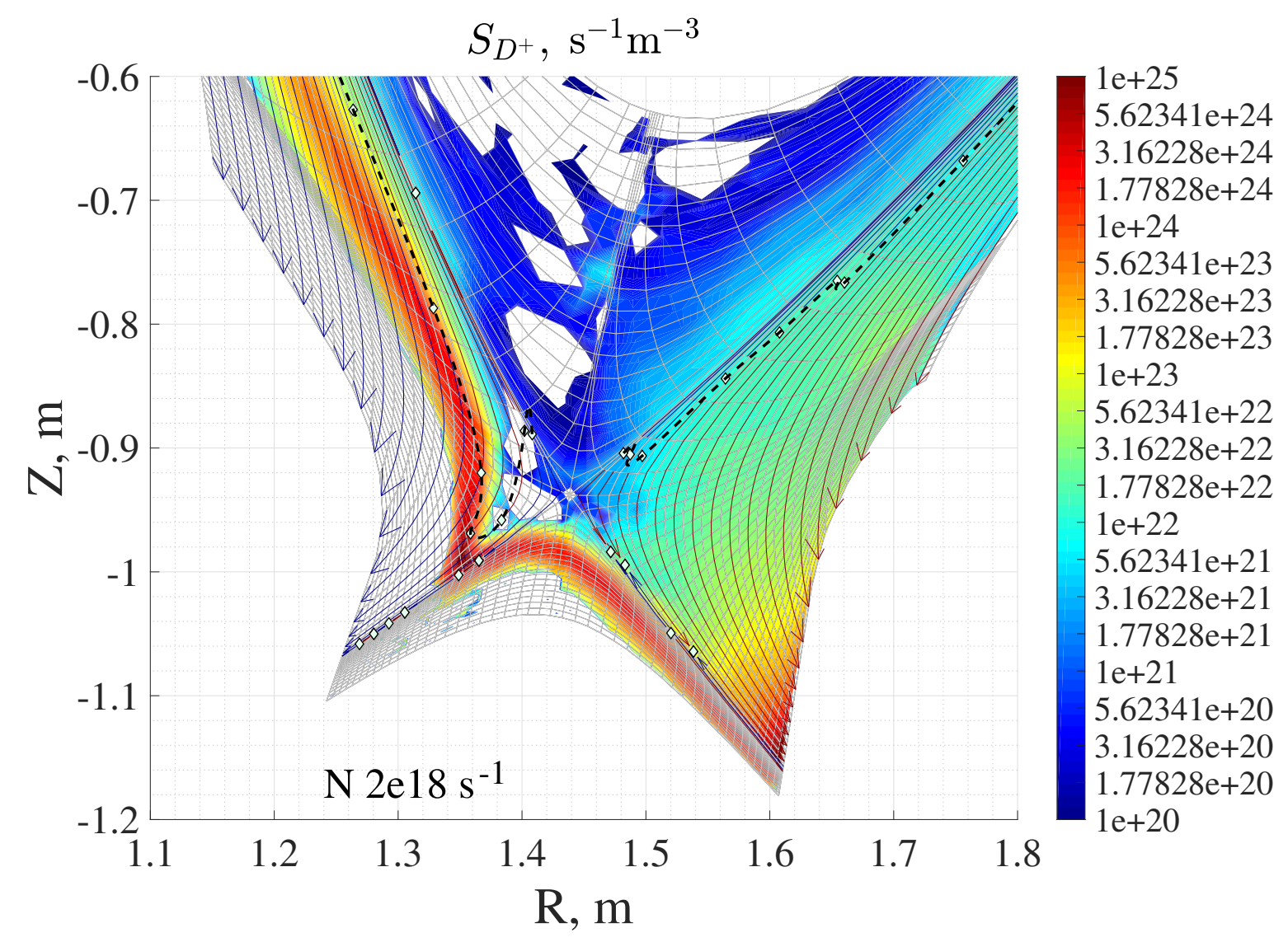

Figure 8. Ionization source strength (contour plot) and direction of net deuterium ion poloidal flow (red and blue arrows). The colorbar indicates the ionization source strength. Red arrows correspond the positive poloidal flux density (with regard to the direction of increasing poloidal coordinate $x$ ), blue arrows - to the negative poloidal flux density. White diamonds represent stagnation points, i.e. places where the poloidal flux is zero. So that each arrow connects either two stagnation points or a stagnation point and a target. Note that ends of arrows which are directed upstream are not seen in this figure, because corresponding stagnation points are located outside the plotted fragment of computational domain. Black dash lines connect stagnation points by spline interpolation excluding points near the separatrix which are affected by radial flows. 

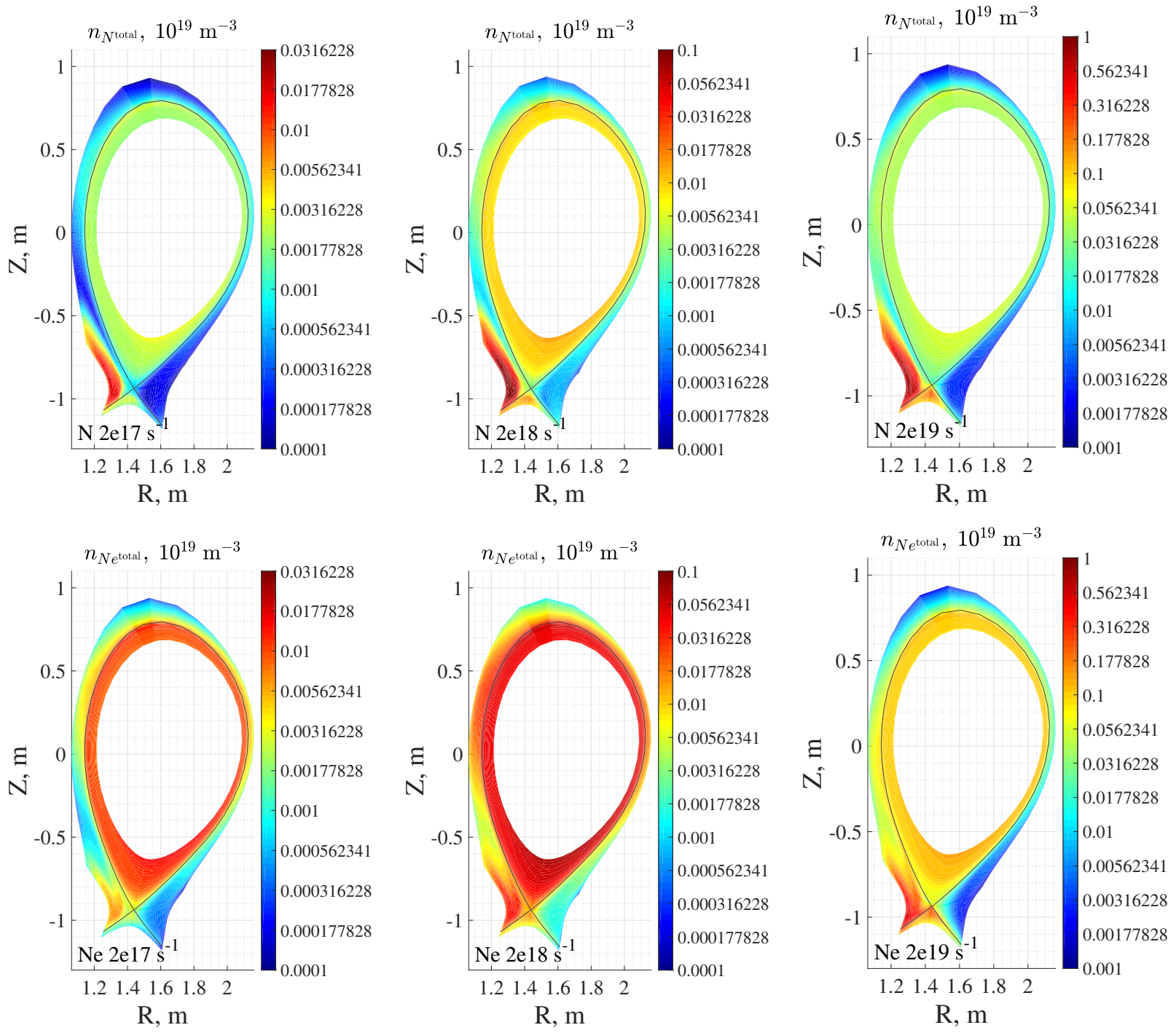

Figure 9. Nitrogen and neon net density for different seeding rates. Mind the different colorbars for different seeding rates!

and to penetrate upstream and further into the core. Correspondingly, the upstream impurity density $n_{\text {imp }}$, as well as the ratio $n_{\mathrm{imp}} / n_{e}$ is bigger for neon than for nitrogen. This qualitatively agrees with experimental observations that the confined region is more strongly affected by the neon than by the nitrogen for an equivalent amount of seeded gas.

\subsection{Details of trace impurity transport}

In this section we focus on the physical mechanisms of impurity leakage or retention and to a lesser extent on the details of the poloidal impurity distribution in the SOL.

The definitive quantity which affects neutral behavior after its ionization is the direction of the impurity ion poloidal velocity at the point where the ionization takes place. The fraction of impurity ions which leaks is equal to the fraction of impurity neutrals which can reach above the stagnation point of the impurity poloidal flow before the ionization.

This is illustrated in Figure 10. The points where the net impurity poloial flux $\Gamma_{p o l}$ imp $=$

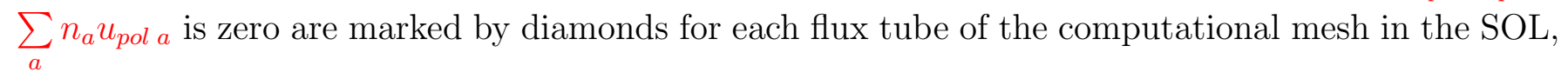
while the ionization source strength is represented by color. These stagnation points on different flux tubes are connencted by dash line. 

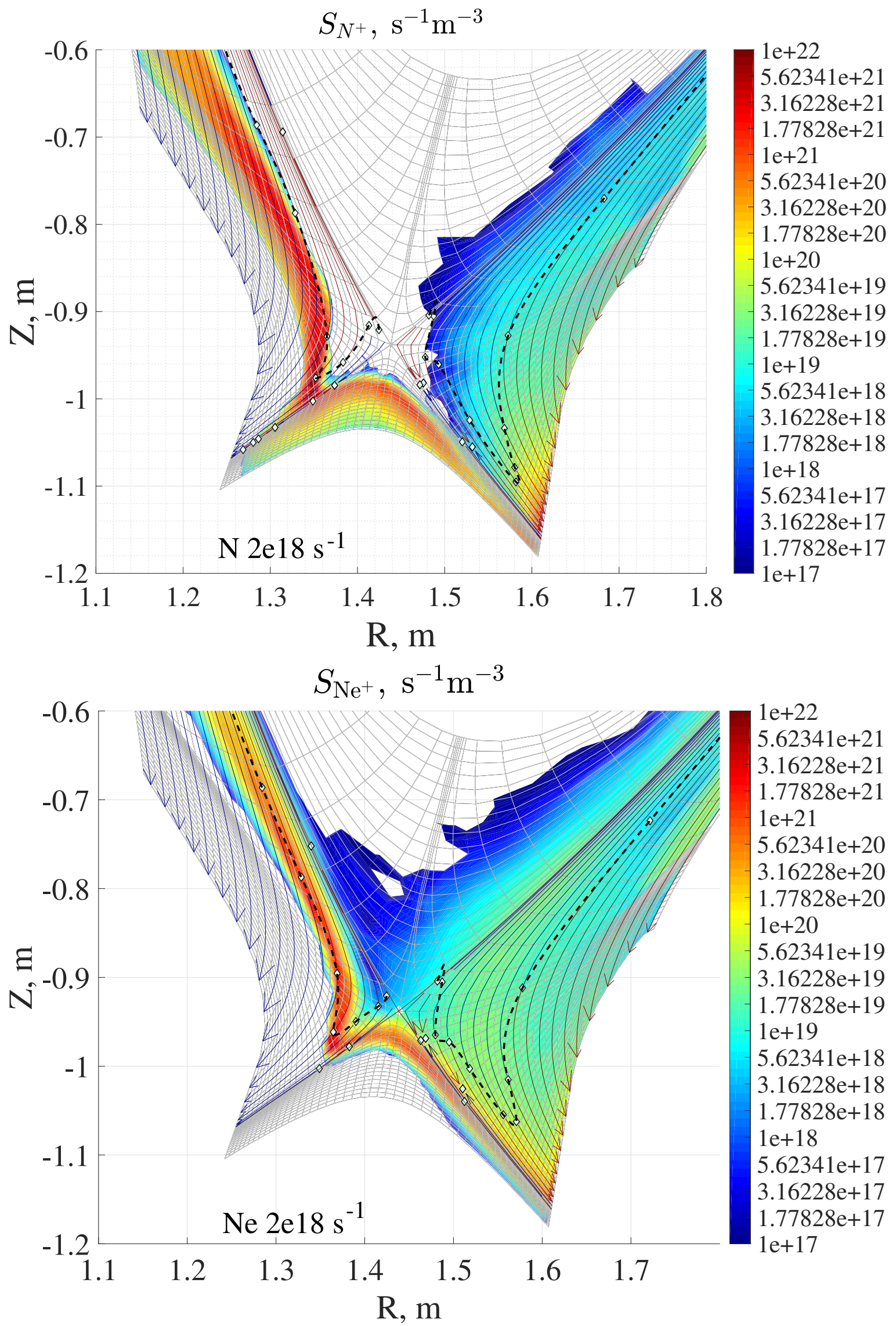

Figure 10. Nitrogen and neon ionization source strength from neutral to first ionization state (contour plot) and the direction of net impurity ion poloidal flow (red and blue arrows). Meanings of colorbar and arrows are the same as in Fig. 8 . 
One can see that starting from some distance from the separatirx radially the flow in the SOL is rather regular in the sense that there is only one stagnation point per flux tube. This is because the neutrals in that region originate either from the recycling on targets (like for the attached outer divertor) or from the volume recombination (like for the detached inner divertor), and both these locations are downstream with regard to the location of the zone where electron temperature becomes big enough for intensive ionization. In the vicinity of the separatrix a notable fraction of neutrals comes from the PFR giving the contribution to the ionization sources along the whole separatrix from the X-point to the target independently of the electron temperature on the nearest flux tubes in the SOL. This ionization source together with $E \times B$ drift across the separatrix [39, 40] produce the irregular structure of ion poloidal flow in the separatrix vicinity, seen in Figures 8,10 both for main ions and impurities. Since neutral source from the recombination both on targets and in the volume is much larger than fueling rate, the perturbation is not so large, and only a zone of less than $1 \mathrm{~cm}$ in width is affected. To make further analysis not too much complex, this zone is excluded from the considerations

Thus in the inner divertor most neutral nitrogen atoms are ionized in the zone where the nitrogen ions poloidal velocity is directed towards the target. This means that most nitrogen ions flow towards the target after the ionization, i.e. nitrogen retains near the inner target.

For neon the situation is different - near the inner target the ionization source remains significant above the stagnation point of the neon poloidal velocity. Consequently, there is a fraction of neon ions which flow upstream after the ionization, i.e. neon tends to leak near the inner target.

Near the outer target the difference between neon and nitrogen is only quantitative: both tend to leak, at least in the near SOL, since there exists a significant ionization source above the poloidal velocity stagnation point for both radiators.

Thus the main result of the present analysis is that whether the impurity leaks or retains is defined by its poloidal velocity profile and by its ionization source profile, but not by the competition between the friction of main ions and the thermal force. This is illustrated once again as 1D plot of poloidal and parallel velocities and ionization source strength along certain flux surface near the inner and outer targets for neon, nitrogen and deuterium, see Figure 11, the seeding rate in these cases is $2 \cdot 10^{18}$ at $/ \mathrm{s}$.

The impurity poloidal velocity to a large extent is defined by their parallel velocity (projected on the poloidal direction) and by the $E \times B$ drift velocity, the $\nabla B$ drift velocity and other small contributions to the poloidal flux (e.g. viscous fluxes) are less important. The difference between $u_{\text {pol }}$ and $u_{\|} B_{\text {pol }} / B$ along certain flux surface may be seen in Figure 11. Surfaces of zero poloidal and parallel velocities for deuterium, nitrogen and neon ions are plotted in Figure 12 — these surfaces are obtained by connecting diamonds in Figures 8 and 10, for the parallel velocity the procedure is the same, the seeding rate for both nitrogen and deuterium in these cases is $2 \cdot 10^{18} \mathrm{at} / \mathrm{s}$. The direction of the poloidal velocity shift with respect to the poloidal projection of parallel velocity corresponds to the positive radial (directed radially outward) electric field in the SOL.

The parallel impurity velocity is defined by the parallel projection of the impurity force balance, where the largest forces are the friction with the main ions and the thermal forces, first the ion-ion one, and then the electron-ion thermal force as well. The impurity pressure gradient and electric force are less important. An example of impurity ion force balance, corresponding to Eq. (2), is plotted in Figures 13 and 14 , again for seeding rate $2 \cdot 10^{18}$ at/s for nitrogen and neon. Note that all plotted forces are computed in the SOLPS-ITER code with full account of all terms appearing in the impurity force balance equation, including viscosity, inertia, ionization and recombination

In the Figure 15 the nitrogen poloidal velocity obtained from Eq. (5) is compared to one 

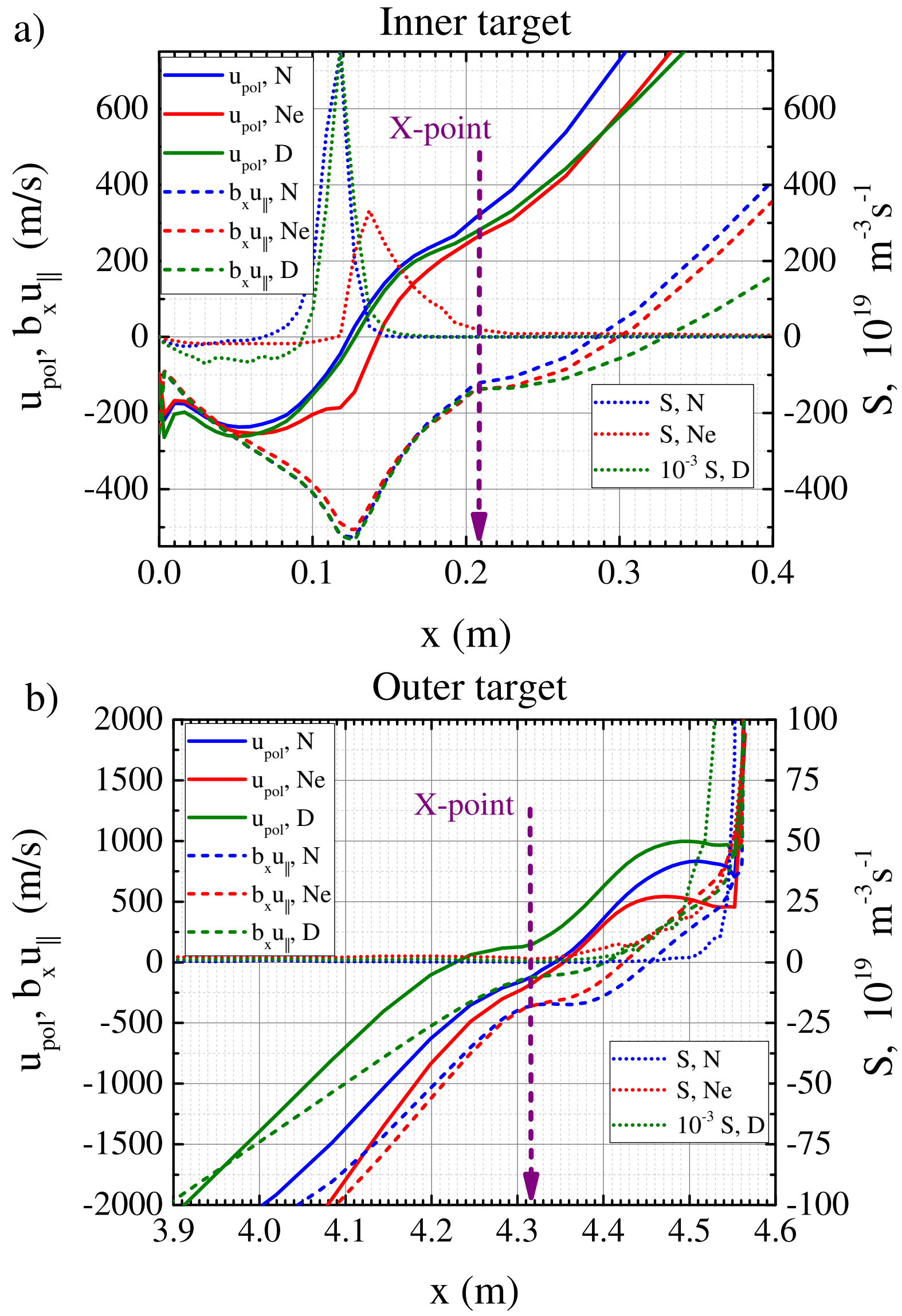

Figure 11. Poloidal velocities, poloidal projections of parallel velocity and ionization sources from neutral state for nitrogen, neon and deuterium in the inner SOL a) at inner target; b) at outer target on the same flux surface, located at $2 \mathrm{~mm}$ from the separatrix as measured at the outer midplane. Poloidal distance $\mathrm{x}$ is measured from the inner target. $b_{x}=B_{\mathrm{pol}} / B$. For impurities instead of $u_{\mathrm{pol}}$ and $b_{x} u_{\|}$plotted are $\left\langle u_{\text {pol imp }}\right\rangle=\Gamma_{\text {pol imp }} / n_{\text {imptotal }}$ and $b_{x}\left\langle u_{\| \text {imp }}\right\rangle$ correspondingly. 


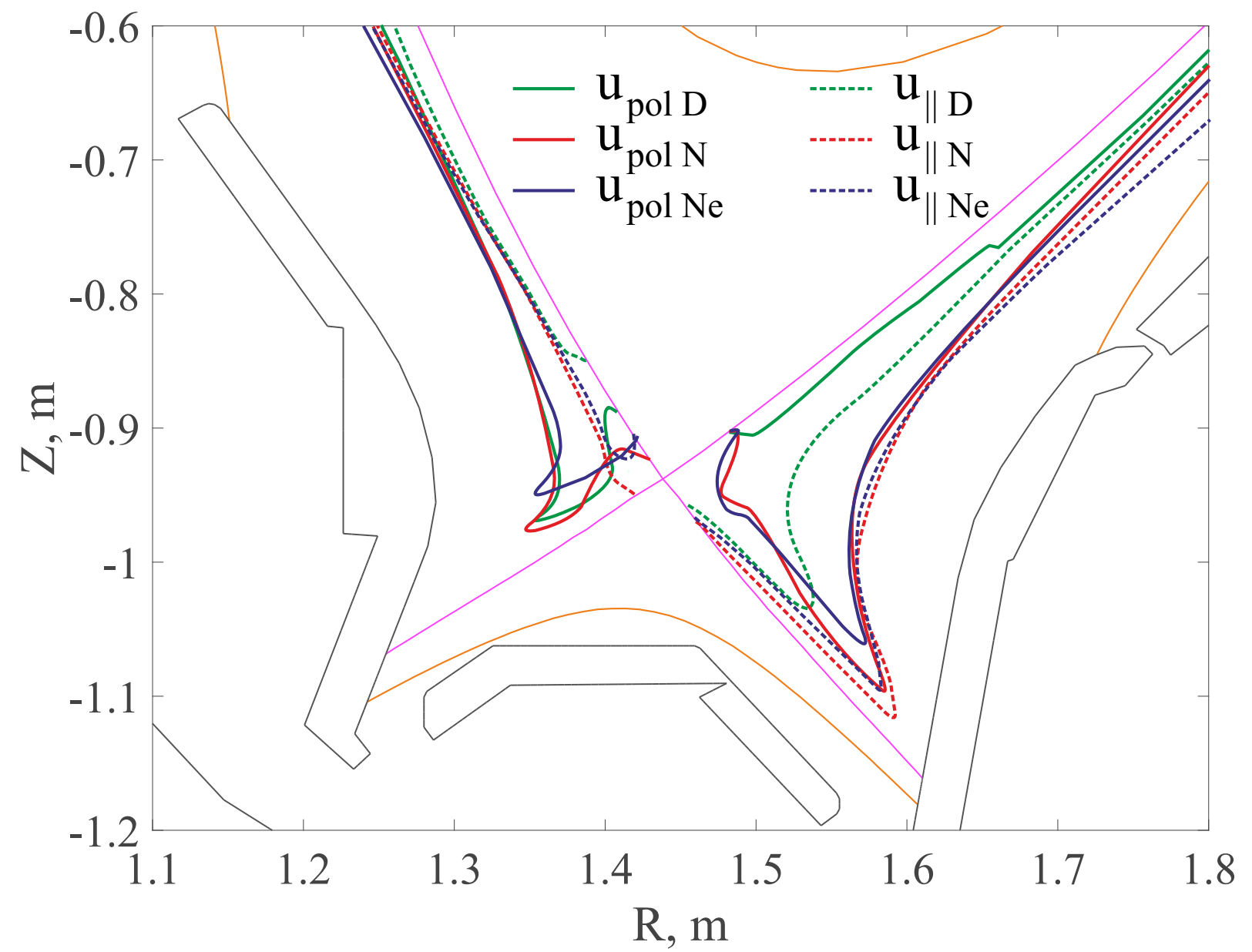

Figure 12. Surfaces of zero poloidal and parallel velocities for deuterium (green color), nitrogen (red color) and neon (blue color) ions in the SOL. Similarly to Fig 11 for impurities instead of $u_{\text {pol }}$ and $b_{x} u_{\|}$plotted are $\left\langle u_{p o l \mathrm{imp}}\right\rangle=\Gamma_{p o l \mathrm{imp}} / n_{\mathrm{imp}}$ total and $\left\langle u_{\| \mathrm{imp}}\right\rangle$. Black lines illustrate in-vessel plasma facing constructions, including divertor plates, magenta line represents the separatrix, orange lines show the boundaries of B2.5 code computational domain.

computed by the SOLPS-ITER code for seeding rate $2 \cdot 10^{18}$ at/s. As a supplement to that in the Figure 15 plotted are poloidal distributions of densities and velocities of nitrogen ionization state species as well as ion and electron temperatures. One can see that the average velocity $\left\langle u_{\| \text {imp }}\right\rangle$, calculated according to Eq. (4), coincides with $u_{\| \text {imp }}$ for most of the individual ionization states (except those which are locally not populated). This coincidence proves the assumption on the validity of the Eq. (5) made in the Section 2.

One can see that the details of the force balance are different at the inner and outer divertors, and, naturally, they change as one moves from the inner to the outer SOL across flux tubes.

Near the outer target, which is attached in our example, and where the plasma is hot enough, the friction between impurity and main ions is compensated mainly by the thermal force. Since the temperature gradient is directed upstream, the thermal force shifts the stagnation point of the impurity parallel flow closer to the target (see Figure 12), and makes it easier for the impurity ions to leak. This shift leads to a corresponding shift of the stagnation point in poloidal flow. As the target is attached, most of the neutrals are ionized very close to its surface (this is better seen in Figure 10), however, some fraction of neutrals can still reach the stagnation point. For neon the effect of its higher ionization potential compared to that of deuterium leads to the leakage together with the effect of the shift of the poloidal stagnation point by the thermal force. For nitrogen these two effects act in opposite directions, however, in our calculations the effect of the stagnation point shift 

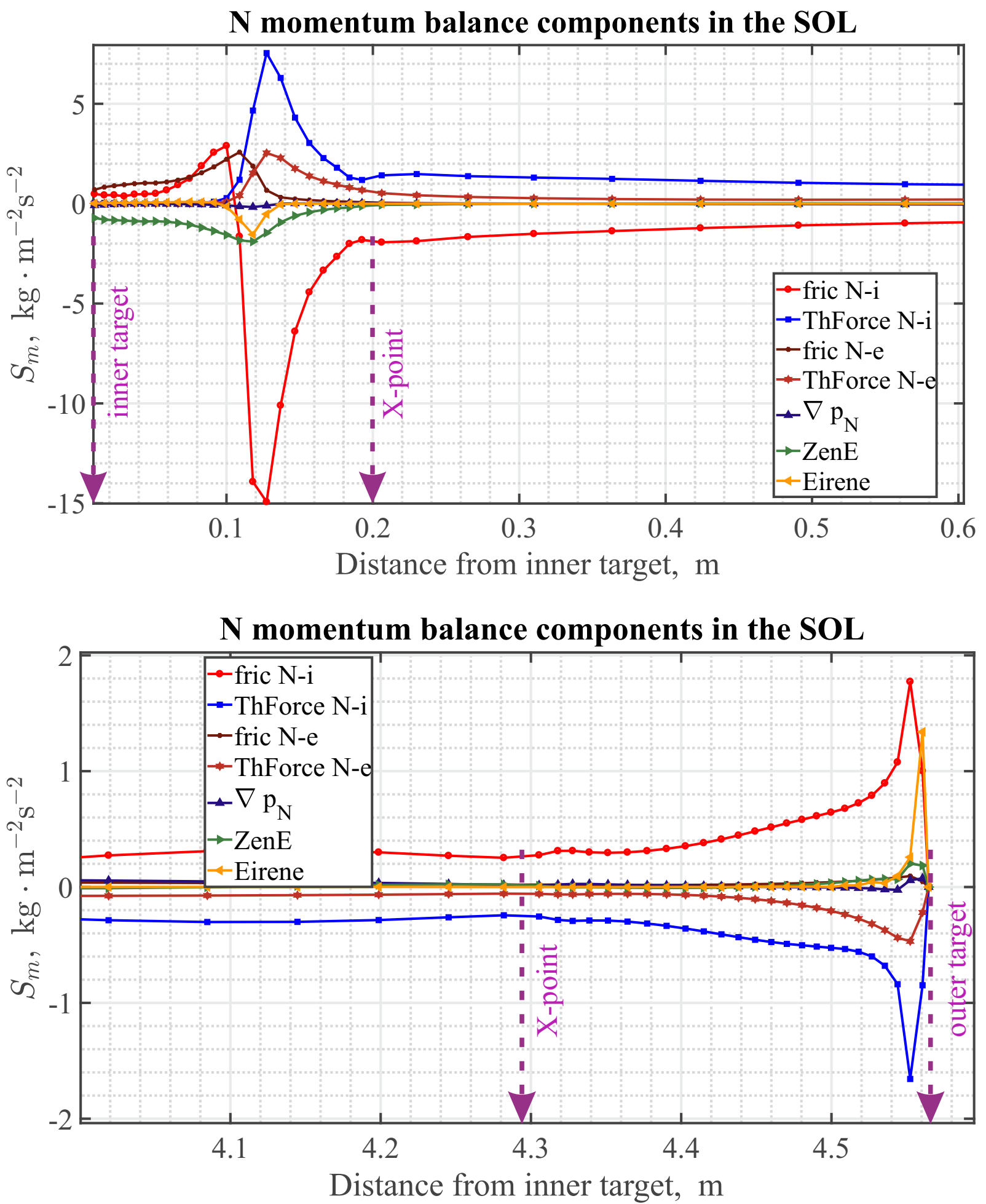

Figure 13. Nitrogen force balance at inner (top graph) and outer (bottom graph) targets in the near SOL (2 mm from the separatrix as measured at the outer midplane) according to the Eq. (2), i.e. forces are summed over all nitrogen ionization states excluding neutrals, for the seeding rate $2 \cdot 10^{18}$ atoms $/ \mathrm{s}$. 

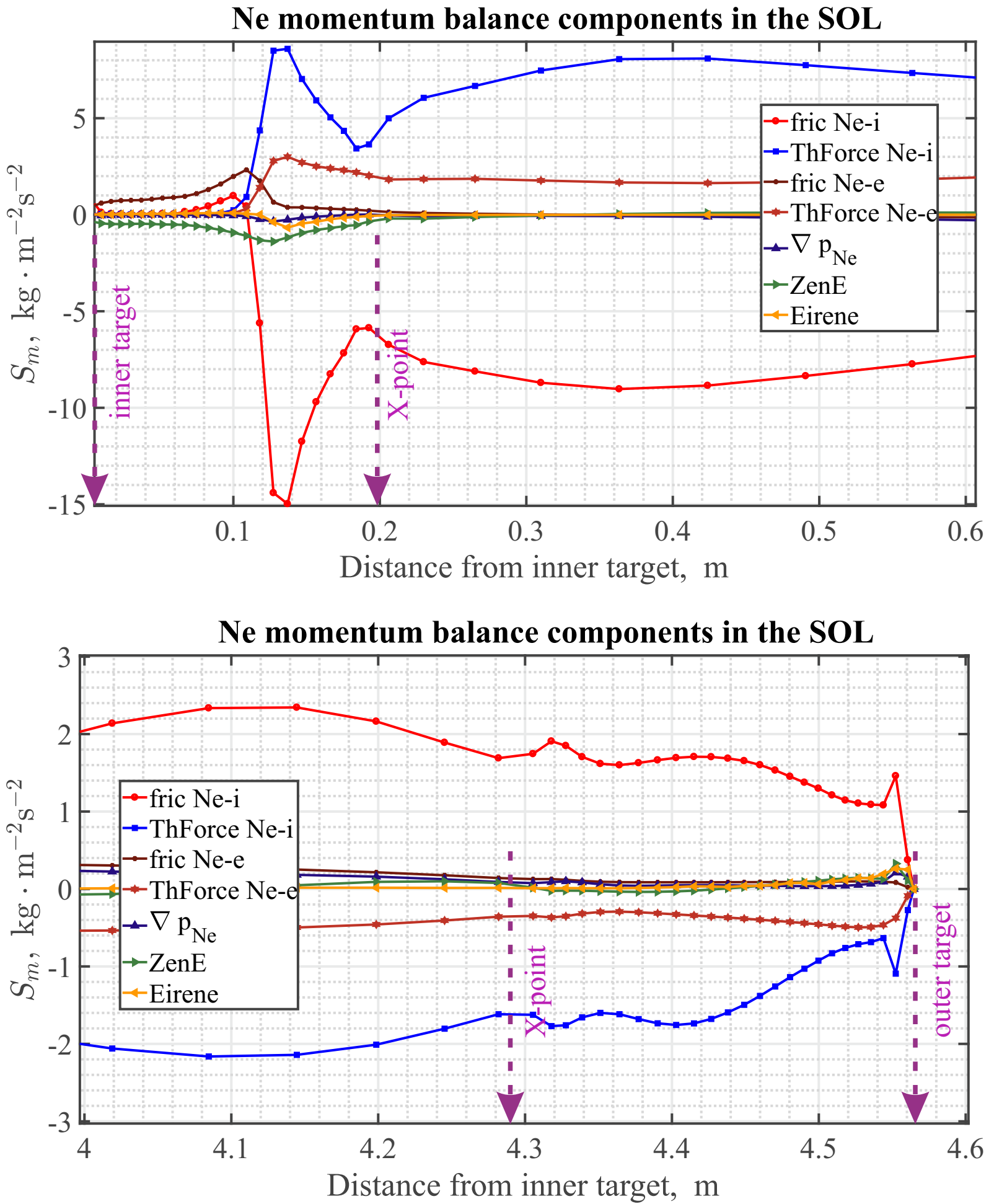

Figure 14. Neon force balance at inner (top graph) and outer targets (bottom graph) in the near SOL (2 mm from the separatrix as measured at the outer midplane) according to the Eq. (2), i.e. forces are summed over all neon ionization states excluding neutrals, for the seeding rate $2 \cdot 10^{18}$ atoms/s. 

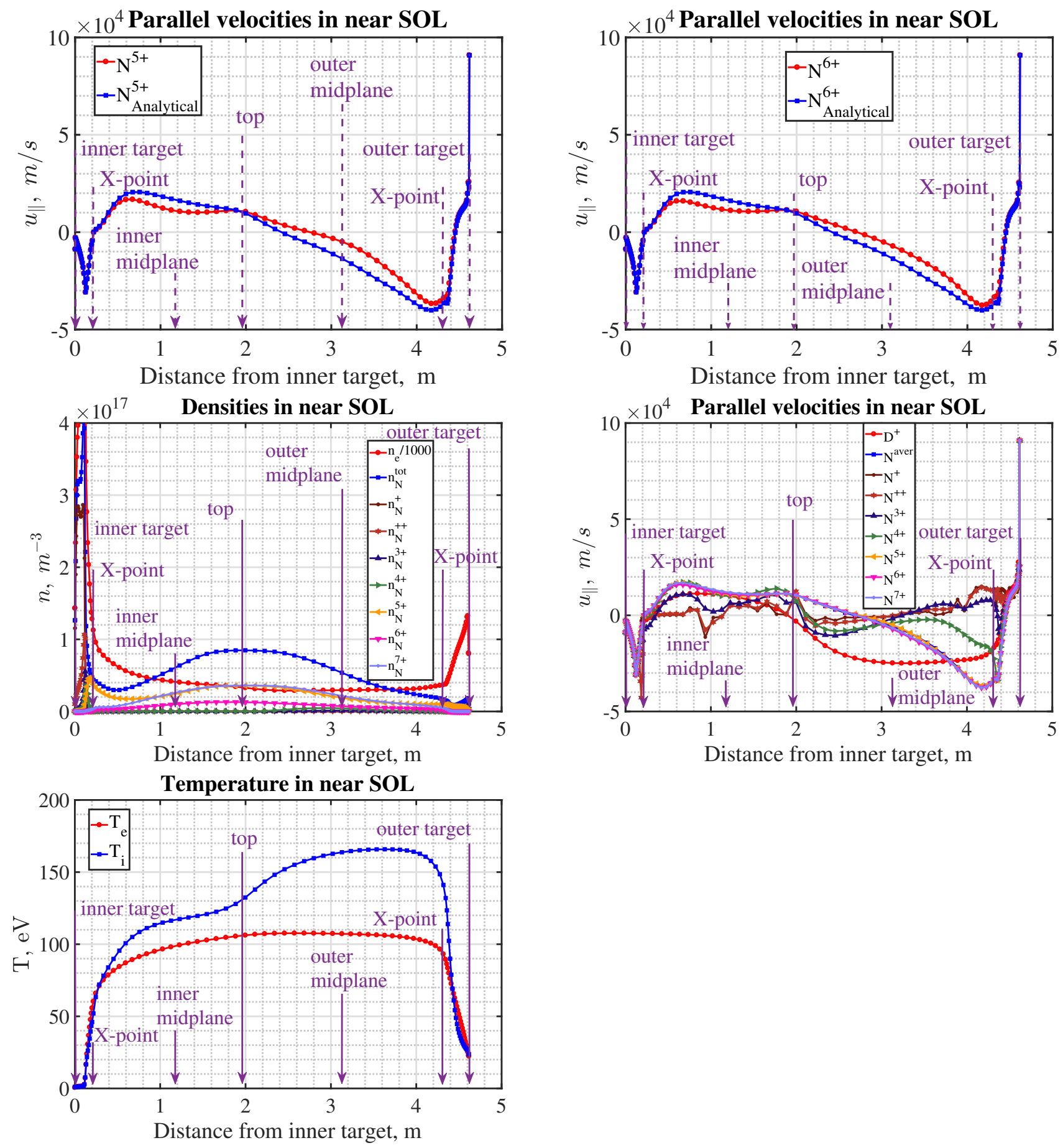

Figure 15. Comparison of nitrogen velocities for species $N^{5+}$ and $N^{6+}$ obtained from the balance of friction and thermal forces (blue lines) to ones computed by SOLPS-ITER code from a full force balance (red lines). For the reference plotted are densities (of electrons, nitrogen ion species and the sum $n_{N_{\text {total }}}$ ), velocities (of main ion, nitrogen ion species and the average $\left\langle u_{\| \text {imp }}\right\rangle$ ) and both ion and electron temperatures along the same flux surface $(2 \mathrm{~mm}$ from the separatrix as measured at the LFS midplane) as the nitrogen force balance plotted in Figure 13 
is stronger, so in spite of faster ionization a significant fraction of nitrogen neutrals can still reach the stagnation point, and the nitrogen at the outer target also leaks. The effect is more pronounced near the strike point (see Figure 10), where a significant fraction of nitrogen neutrals ionizes after crossing the stagnation point of poloidal flow. Denoting this fraction as $f$, one can mention that in our calculations $f_{\mathrm{Ne}}>f_{\mathrm{N}}>f_{\mathrm{D}}$. Consequently, one would expect that the same inequality is true for the ratios of upstream to the divertor densities, and the opposite for compressions, namely $C_{\mathrm{Ne}}<C_{\mathrm{N}}<C_{\mathrm{D}}$, and it is indeed the case in the simulations, if one uses the ratio $n^{\text {div }} / n^{\text {up }}$ as a measure of compression and substitutes the outer midplane density (summed over all ionization states) for $n^{\text {up }}$ and takes the divertor density at the same flux tube (again the sum over all ionization states), see Figure 27 below.

Near the detached inner target there is a zone of cold dense plasma, where the ion-ion collision frequency is very large, and the 2-dimensional temperature profile is almost flat. In these conditions any significant difference between main ion and impurity parallel velocities leads to a very large friction force which cannot be compensated by any other force, therefore the main ion and impurity velocities coincide. Then the two largest contributors are the friction between impurity ions and electrons and the electric force, like in a parallel projection of the electron force balance. The thermal force becomes significant even further upstream than the place where the temperature starts to rise and a strong ionization front appears. Inside this zone of the ionization the main ion density significantly increases making the friction force stronger, so that the parallel velocities of main ions and impurities coincide up to the X-point, i.e. further than the ionization front, in spite of a presence of the thermal force caused by the strong temperature gradient, see Figure 11 a). Thus the effect of the parallel velocity stagnation point shift by the thermal force is not important near the inner target in our simulations.

In this situation the impurity ionization rate becomes the most relevant quantity. If it is smaller than the ionization rate of the main ion, then most impurity neutrals ionize closer to the target than the main ions do, i.e. before reaching the common stagnation point of poloidal velocity profile, and therefore the impurity is retained in the divertor. More traditionally, one usually considers the ionization potential instead of the ionization rate. However, in the case of nitrogen, which has a ionization potential larger than that of deuterium $(14.6$ vs $13.6 \mathrm{eV})$, its ionization rate $\left\langle\sigma_{\text {ion }} v\right\rangle$, is nevertheless about 2 times bigger than for deuterium for the temperature range of interest. This allows for nitrogen neutrals to ionize closer (or at least not further) from the target than the deuterium neutrals do (see Figure 11 a)), again before reaching the common stagnation point, so the nitrogen is retained.

In the opposite case (like for neon) the impurity neutrals can penetrate through the zone of main ion ionization and reach past their stagnation point. One can see from Figure 11 a)) that the neon ionization source is located further upstream than the deuterium and nitrogen ones. Thus after the ionization such an impurity is dragged away from the target, i.e. the impurity leaks.

The leaked ions in a steady state should return back to the divertor, so that there should exist closed impurity flow loops, which consist of parallel flows, perpendicular flows and flows of neutrals, with account of neutral-ion and ion-neutral transformations due to ionization and recombination. Such an impurity flow in the SOL and the PFR calculated for nitrogen and neon are shown in Figures 16 and 17. The analysis of parallel and perpendicular (with respect to the B-field) impurity transport results in that the friction force and thermal force almost compensate each other everywhere throughout the SOL, and that the impurity parallel velocity may be found from this balance. Example of such a comparison between the nitrogen parallel velocities obtained from Eq (5) and ones computed from the full force balance in the SOLPS-ITER code is presented in Figure 15. The divergent part 


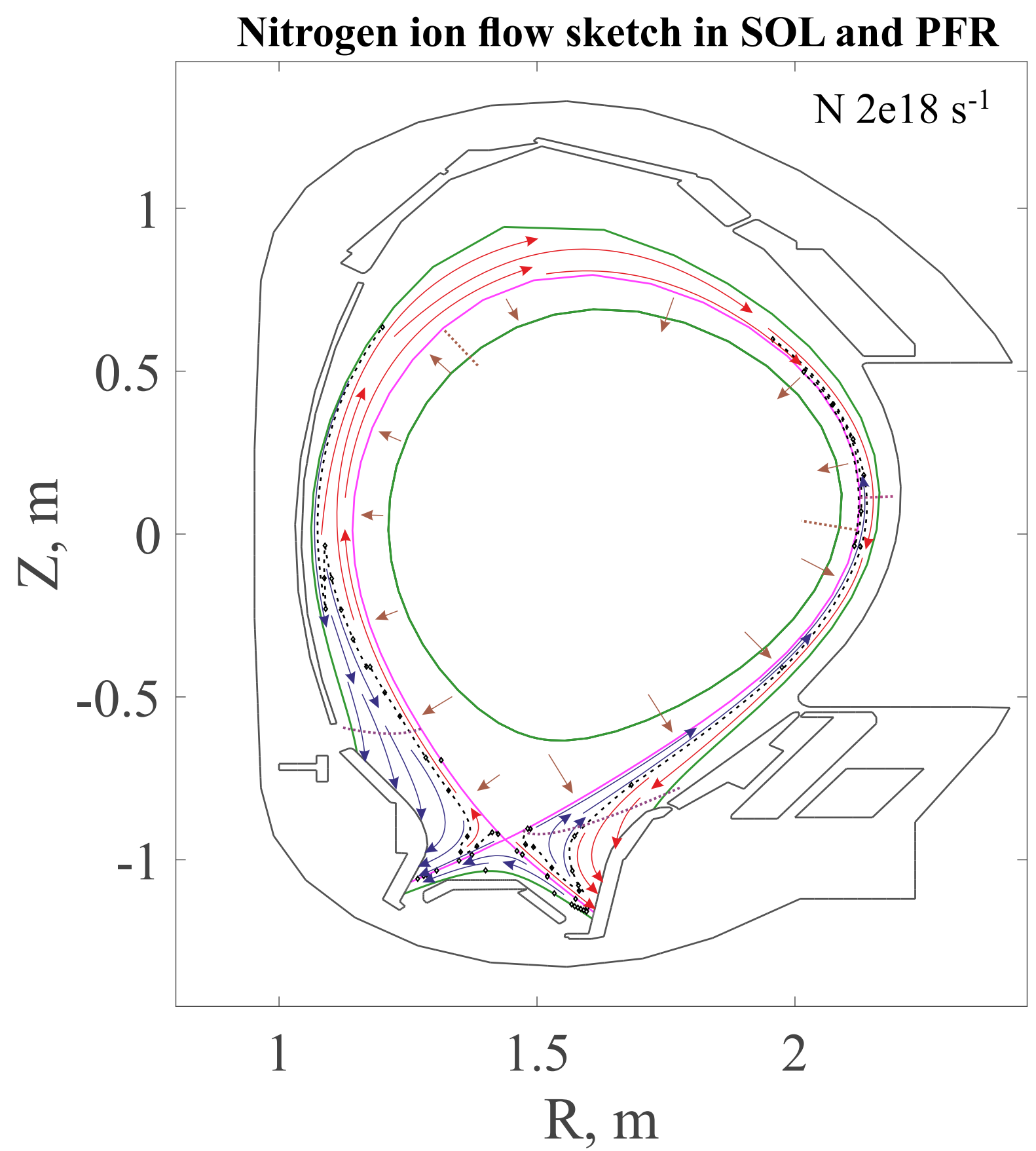

Figure 16. Nitrogen poloidal flow $\left(\Gamma_{p o l} \mathrm{~N}\right)$ sketch in SOL and PFR for the N seeding rate $2 \cdot 10^{18}$ atoms/s in AUG. Red arrows represent positive flow, blue ones - negative (with respect to the direction of x-coordinate growth). Black dash curves show the locations where poloidal velocity is zero. Violet dot lines across the SOL show the points where the net poloidal flux through the SOL changes its sign. Brown arrows show the direction of radial (diffusive plus $\nabla B$ drift) flux across the separatrix which is plotted in magenta. Brown dashed lines across the core region show locations where radial flux density changes its sign. Length of arrows and their density do not reflect the value of flux - arrows just show flow direction. 
Neon ion flow sketch in SOL and PFR

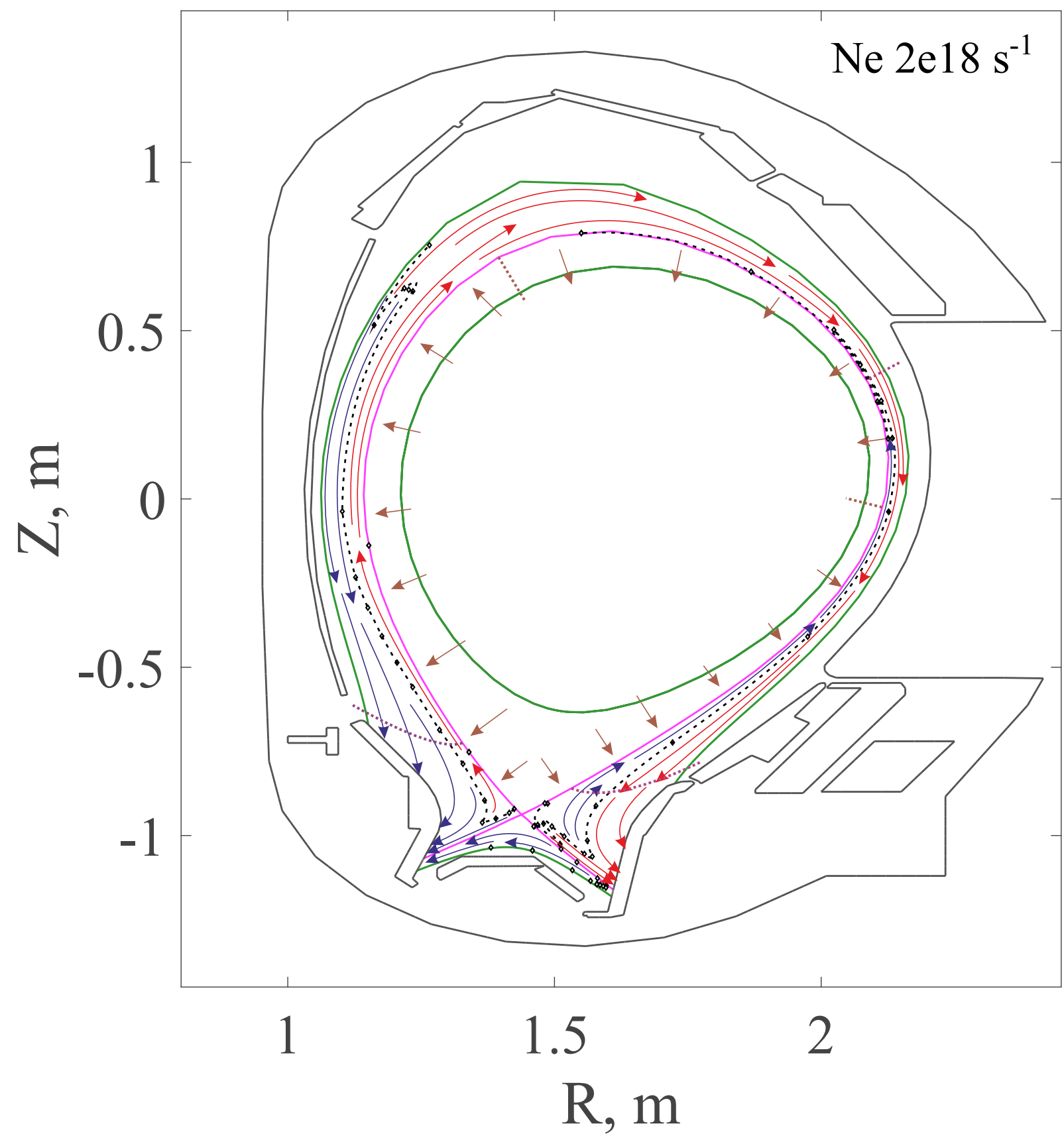

Figure 17. Neon poloidal flow $\left(\Gamma_{\text {pol Ne}}\right)$ sketch in SOL and PFR for the Ne seeding rate $2 \cdot 10^{18}$ atoms/s in AUG. Meanings of color arrows, dash and dot lines are the same as in Fig. 16.

of poloidal impurity flux together with the ionization/recombination source is compensated mainly by the radial diffusion, and the local impurity density is defined from this condition.

Thus we come to a conclusion that the parallel flow pattern of the main ions plays a determinative role in the impurity transport in the divertor region as well as in upstream, since impurities are dragged by the main ions due to the strong friction defined by $u_{\| D}$. The details of main ion transport in the SOL and the role of drifts in it is discussed in the next subsection. 


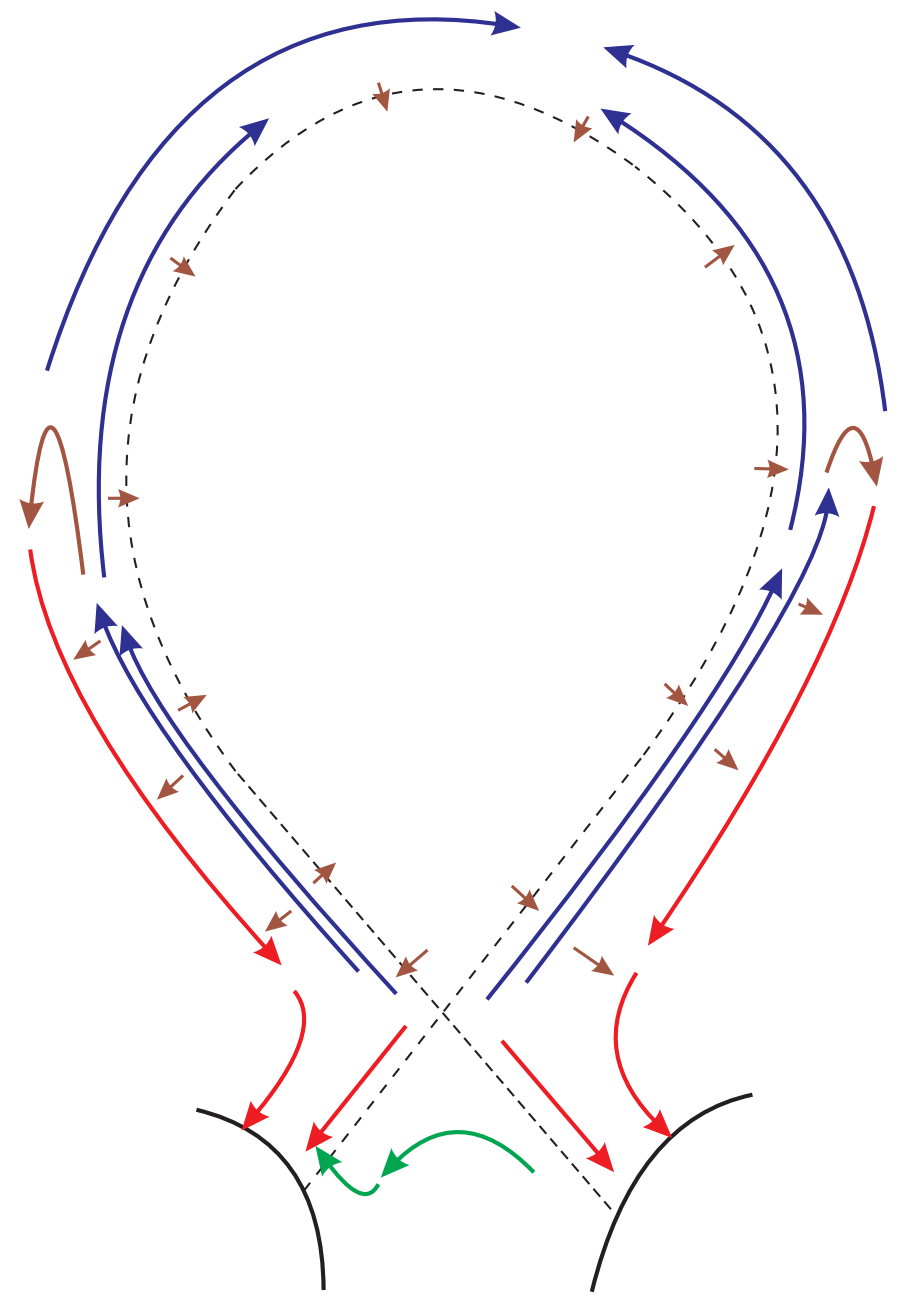

Figure 18. Schematic picture of the main ion poloidal flow pattern in the SOL and in the PFR, which corresponds to the impurity seeding rate $2 \cdot 10^{18} \mathrm{~s}^{-1}$. Red arrows represent poloidal flows towards the divertors, blue arrows - out from divertors, brown arrows show a direction of radial fluxes (sum of $\nabla B$ drift and diffusive ones), green arrows demonstrate the $E \times B$ flow direction in the PFR.

\subsection{Role of drifts in the main ion and impurity flow pattern}

According to the continuity equation, the parallel flow of main ions closes the divergent part of the $\nabla B$ and $E \times B$ drifts and of the anomalous diffusion flow across the B-field, and compensates the particle sources and sinks due to ionization and recombination. Above the X-point in the near SOL the main contribution to the parallel flow in AUG is the Pfirsch-Schlüter flow, which is directed from the X-point towards the top of the tokamak (in the geometry considered the ion $\nabla B$-drift is directed downwards). Below the X-point the much larger fluxes are directed towards the targets providing the recycling.

However, the ion motion after it is born from a neutral is defined by the poloidal flux and its stagnation points, which are different from the parallel ones. The poloidal ion flow is defined by the poloidal projection of the parallel flow, the poloidal components of the $E \times B$ and $\nabla B$ drifts and to a lesser extent by the anomalous diffusion $-D^{\mathrm{AN}} \nabla n$. These stagnation points are illustrated in Figure 12. Note that most ions are ionized closer to the target than the location of the poloidal flux stagnation point (see Figure 11) to make the recycling flow to be much bigger than the upstream leakage flow.

Further (from the separatrix) in the SOL the stagnation point of the main ion poloidal flow 

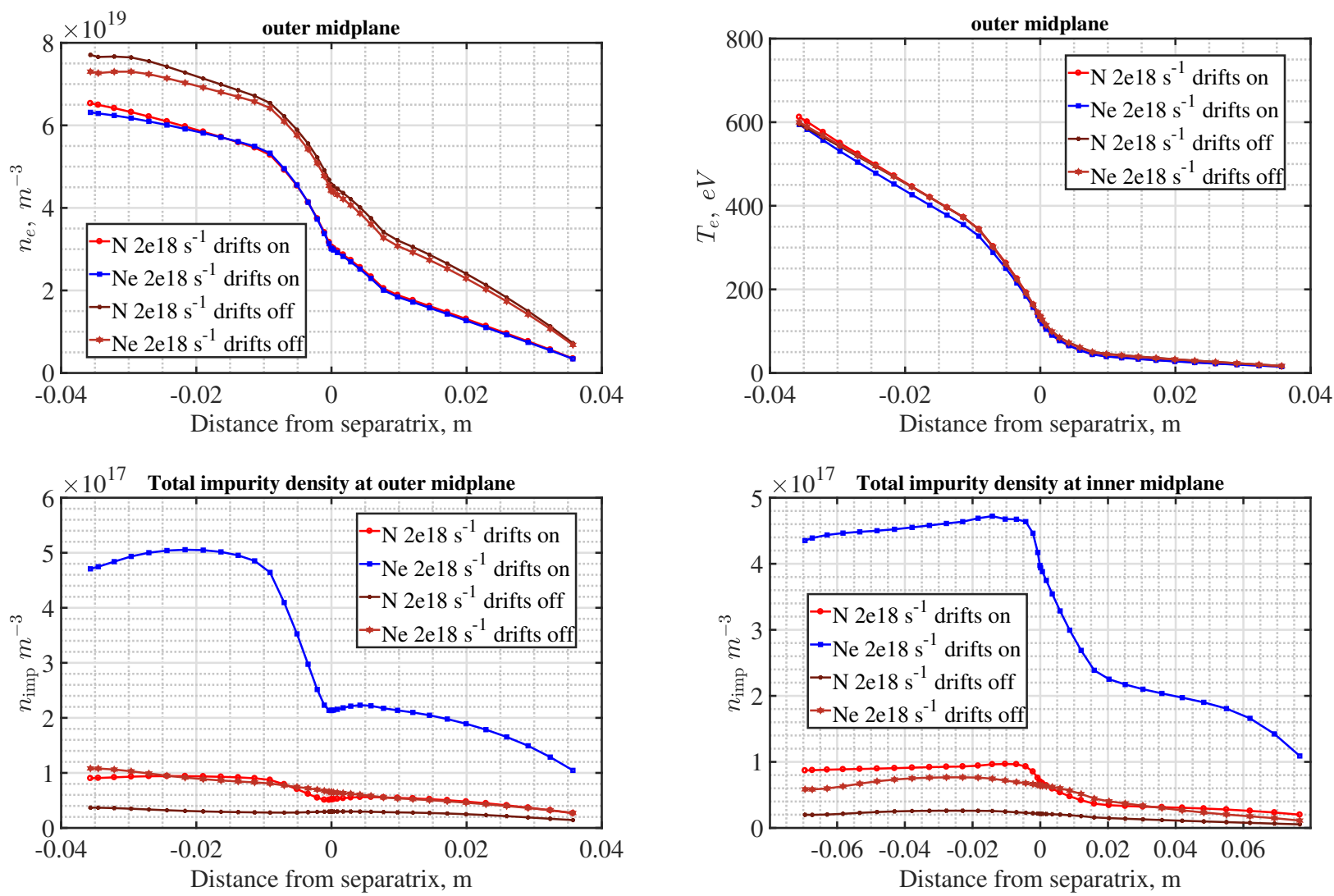

Figure 19. Outer midplane profiles of electron density, electron temperature and impurity density (sum over all charged states) for runs with drift turned on (normal) and off. Impurity density is plotted at inner midplane too.

profile is shifted upstream because of two reasons. First, the strength of the Pfirsch-Schlüter fluxes decreases in the far SOL. Second, the relatively small fraction of particles born due to ionization in the near SOL and carried away towards the midplane by the upstream flow (see Figure 8) should return back to the divertor. This return flow is provided by the radial diffusion towards the far SOL and by the parallel flow towards the targets, where particles recombine. Then the loop is closed by neutral flow from the far SOL to the ionization zone in the near SOL, see Figure 18. Such flow reversal loops are seen in the simulation results at both inner and outer targets.

It is worth mentioning that the flow reversal phenomena was observed in several other 2D transport simulations without drifts [19], where some flow above the region of maximal ionization was seen, however much smaller than the Pfirsch-Schlüter one, see below.

Another important contribution to the main ion flow pattern is the $E \times B$ drift in the Private Flux Region (PFR), which grabs all ions born in the PFR due to ionization and drags them towards the SOL on the High Field Side (HFS) [39, 40, 41]. The PFR is filled by neutrals either by the gas puff or by the neutrals trajectories from the point of recombination in the SOL volume or at the targets. A neutral flux equivalent to the puffing rate is directed from the divertor plasma towards the subdivertor volume, where these particles are pumped away.

Note that the puffing/pumping flux is usually an order of magnitude smaller than the recycling flux, which is equal to the ion sink due to the recombination either in the divertor volume or at the targets. A small imbalance between ion sources and sinks in the HFS and LFS divertors may be compensated by a net parallel flux in the SOL through the top of the machine (also including the poloidally dependent radial fluxes to and from the core). In our simulation such a net SOL flux is small, even when compared to the puffing rate. 

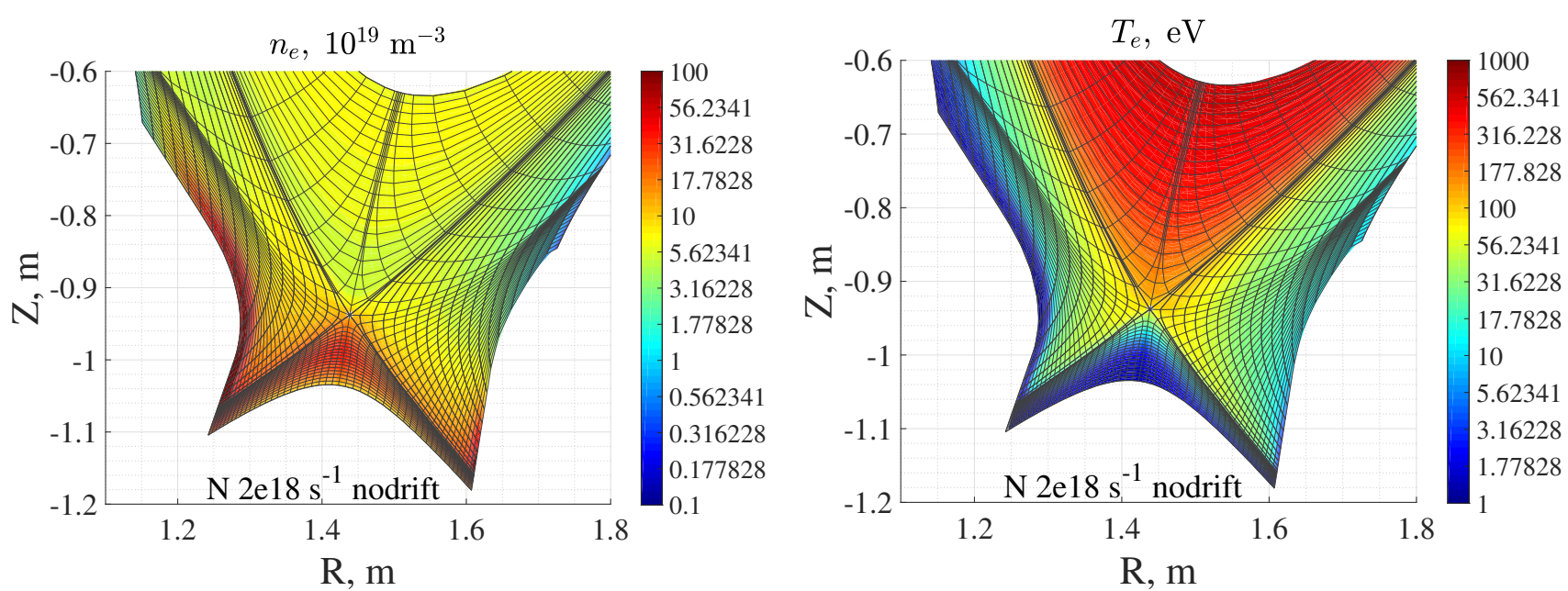

Figure 20. 2D contour plots of electron density and temperature in the vicinity of X-point and targets for runs with drift turned off. Compare to Figure 7 and keep in mind different upstream density.

The parallel fluxes of impurities are defined by friction of main ions (with account of thermal force), and their poloidal velocity is to the large extent the sum of poloidal $E \times B$ velocity and poloidal projection of parallel velocity. This means that impurity poloidal flows are prescribed by main ion flows, i.e. by main ion velocity and by ion and electron temperatures, which are almost not affected by trace impurities. So that the only way for impurities to make their fluxes divergent free is to adjust their density in such a way that radial diffusive fluxes will compensate the divergent part of poloidal flow. Also, like for main ions, the important role is played by radial $E \times B$ drift across the separatrix below the X-point. These drifts are directed from outer divertor through the PFR to the inner divertor, and are partially compensated by net poloidal flow in the SOL through the top of the machine, see Figures 16 and 17.

To investigate the role of drifts and currents, 4 additional scenarios with nitrogen and neon seeding rates set to $2 \cdot 10^{17}$ and $2 \cdot 10^{18}$ atoms per second were calculated. In these cases the $E \times B$ and $\nabla B$ drifts, and corresponding currents, including also the viscosity currents were turned off (i.e. artificially multiplied by zero) thus forcing the SOLPS-ITER code to work in a mode similar to the older version SOLPS 4.3, while the remaining parameters, including anomalous transport, boundary conditions of prescribed deuterium NBI particle influx and prescribed power in electron and ion channels at the core boundary of the computational domain, are kept unchanged.

The results are presented in Figures 19, 20, 21, 22, 23, where some significant differences may be seen. First, the divertor asymmetry, which is clearly seen with drifts turned on, becomes much less pronounced without drifts. The HFSHD region disappears, charged particles migrate to the outer divertor region causing a transition to semi-detached operation there. This result confirms the essential role of the PFR $E \times B$ drift flux in the HFSHD formation, mentioned in [39, 41]. It is worth mentioning that in the simulations without drifts the upstream density increases, while the net particle and heat fluxes through the core remain unchanged. This is the expected result of the simulations without drifts, since in this case no drift contribution to the convective heat fluxes exist, and higher density is required to provide the same convective heat flux by radial particle diffusion.

The poloidal flow pattern of the main ions changed significantly both in the SOL and in the PFR, because the $E \times B$ and Pfirsch-Schlüter fluxes, which play an important role, disappear. Absence of these fluxes also affects the impurity poloidal fluxes and a poloidal distribution of the radial fluxes crossing the separatrix both for main ions and for the impurity. As a result, the impurity distribution 

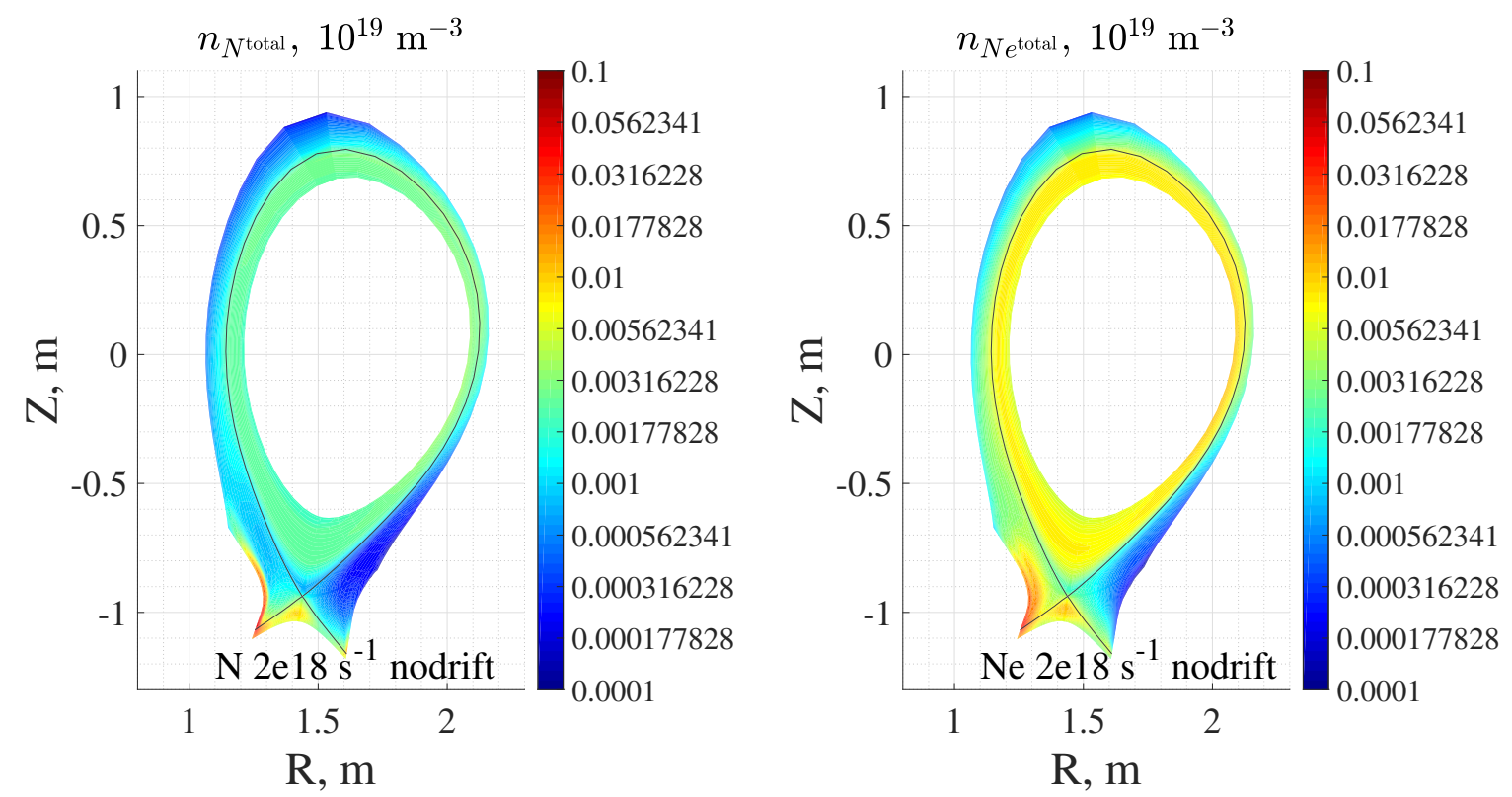

Figure 21. Nitrogen and neon net density with drifts turned off. Compare to Figure 9 .

drastically changes, in particular, the core impurity density decreases fivefold as drifts are turned off. Note that the core impurity concentration decreases even more, since the main ion density increases in the cases without drifts.

Summarizing what is said above, important effects of drifts are following: i) presence of PfirshSchlüter parallel flows of main ions, which affects the parallel velocity of main ions; ii) contribution to the poloidal velocity of impurities; iii) drag of ions from outer divertor to the inner one through the PFR, which gives rise to the divertor assymetry [39, 40] and High Field Side High Density [17]; iv) contribution to the boundary conditions on targets; v) radial neoclassical impurity transport in the confined region (not discussed in present paper). Thus one can conclude that the full account of drifts and currents is essential for the correct computation of divertor working regime and impurity distribution.

\subsection{On the impurity density distribution in the $S O L$}

As it was mentioned above, impurities are coupled to main ions through the strong friction, and the thermal force provides a difference between main ions and impurity parallel velocities. As a result, poloidal flows are coupled similarly to parallel ones, since they differ mainly by the $E \times B$ drift velocity common for all ions. Thus the reversal flows through the far SOL also exist for impurities like for main ions to return leaked impurities back to the targets - these flows are simply coupled by friction. This means that in the far SOL no stagnation point exists in the target vicinity, so that after the ionization both main ion and impurity flows are directed towards the targets. However, the absolute values of these upstream flows in the near SOL and reversal flows in the far SOL are bigger for neon than for nitrogen, both at the HFS and the LFS, since the fraction of ions which leaks is bigger for neon.

This results in higher upstream density for neon than for nitrogen.

Again, like for the main ions, there may exist an imbalance between impurity sources and sinks near the targets, which may be compensated by the net poloidal flow through the SOL. This poloidal flow, together with the reversal flows, is provided mainly by the friction with the main ions with some modification due to the thermal force, and almost independent of the poloidal pressure gradient. 

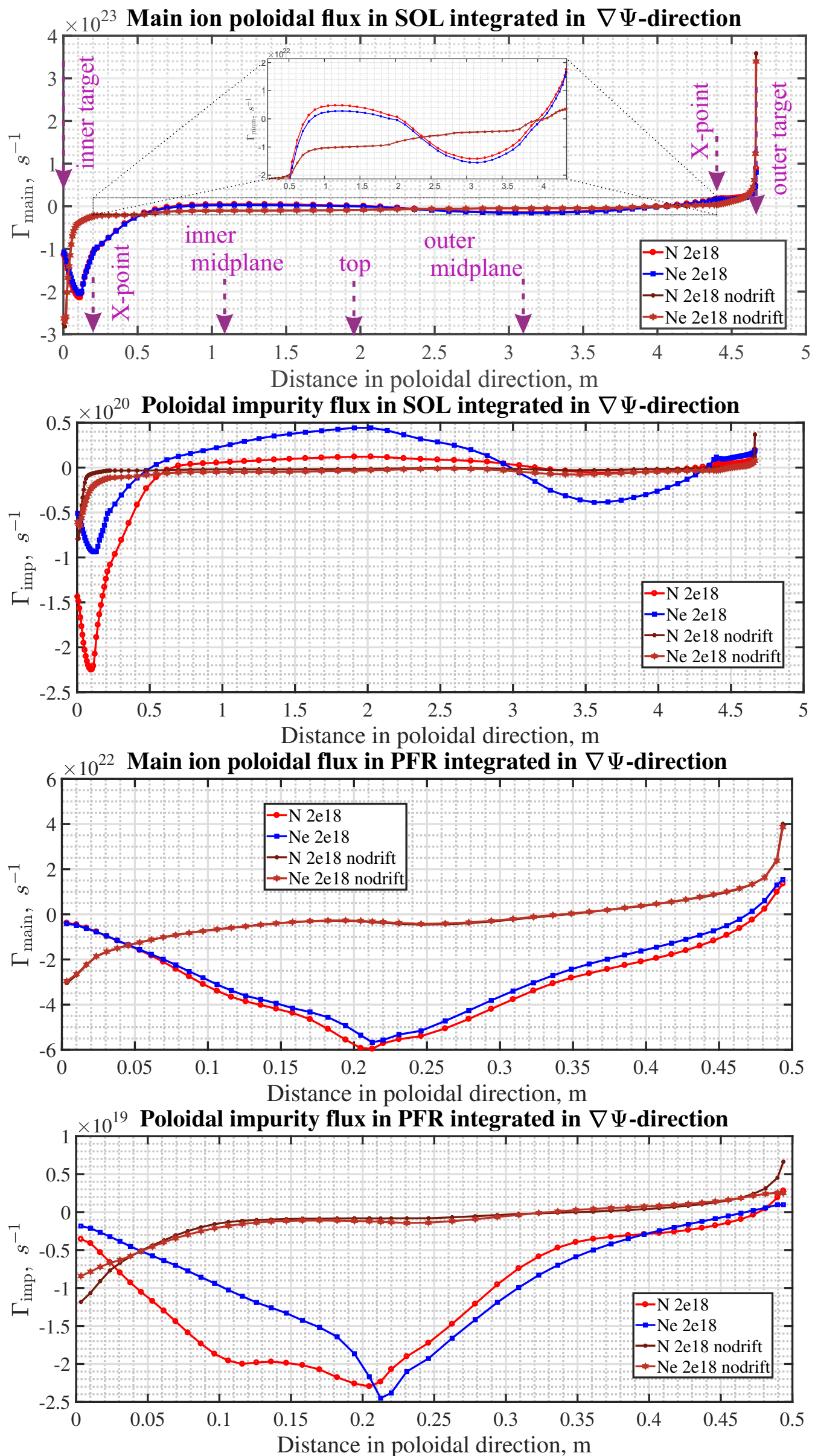

Figure 22. Main ion and impurity net poloidal fluxes (for nitrogen or neon they are $\Gamma_{p o l \mathrm{~N}}$ and $\left.\Gamma_{p o l ~ N e}\right)$ through the SOL and PFR for cases with drifts turned on and off. $\Psi$ denotes poloidal magnetic flux, $\nabla \Psi$ defines the direction across flux surfaces. In the first viewgraph the zone from the $\mathrm{X}$-point at HFS through inner midplane, machine top and outer midplane back to the X-point at LFS is zoomed in to better show the Pfirsch-Schlüter modulation for the cases with drifts turned on. 

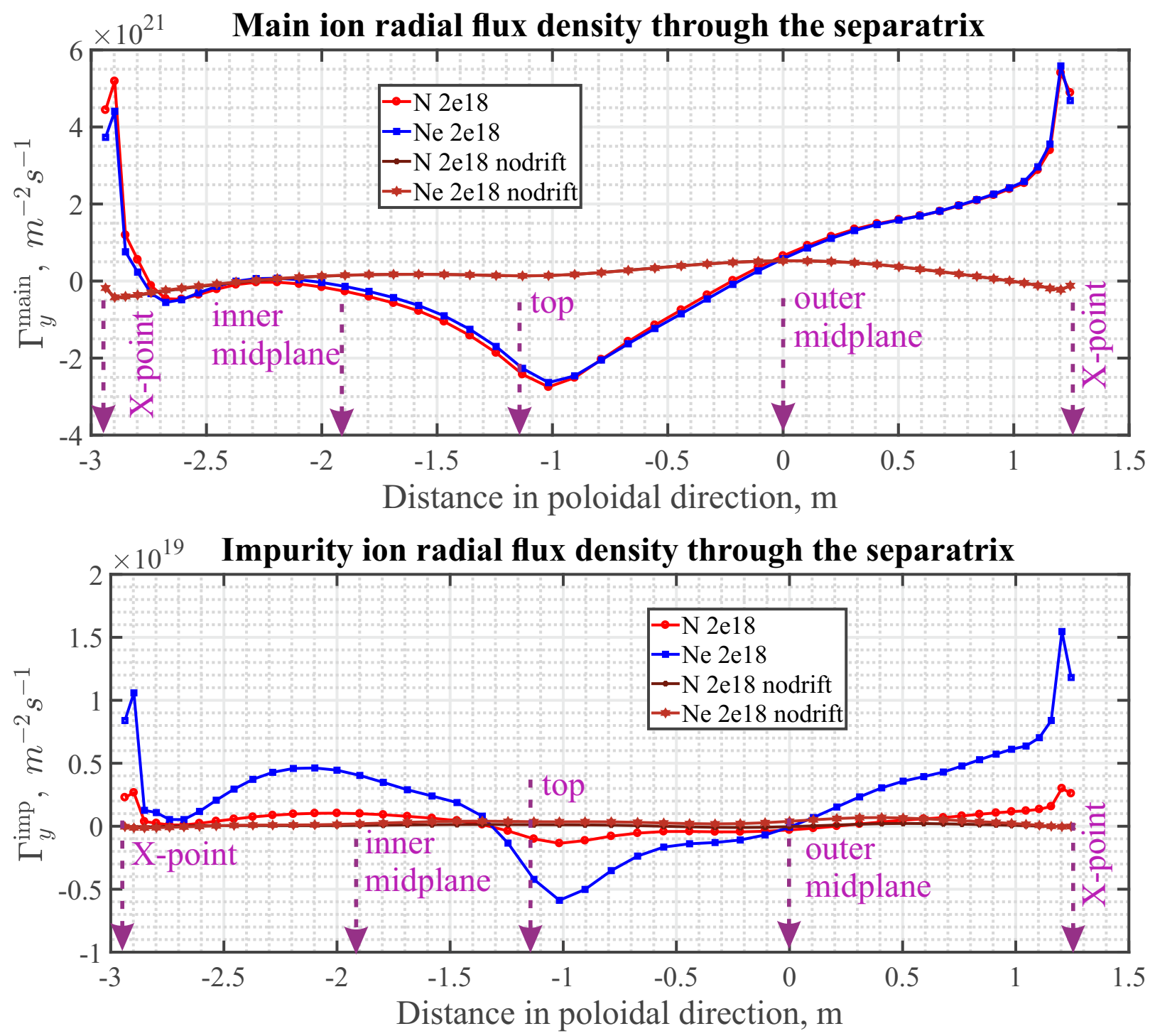

Figure 23. Main ion and impurity (nitrogen or neon, sum over all charged states) radial flux density across the separatrix from the X-point at HFS through the machine top to the X-point at LFS for cases with drifts turned on and off. Origin on the abscissa axis corresponds to the equatorial midplane, LFS.

Thus the poloidal distribution of the impurity density in the SOL above the X-point is defined by the requirement to close the divergent part of poloidal impurity fluxes, i.e. to provide the radial diffusive fluxes to those zones of the SOL where the friction with the main ions would drag impurities in the required direction. If such zones in the SOL do not exist, the net impurity ion flux from one target to another may flow partially through the core, and radial impurity fluxes to and from the core at different poloidal locations are also driven by diffusion. As a result, the poloidal profile of the impurity radial flux through the separatrix may have a different shape than the one for the main ions.

Consequently, we come to the conclusion that the local impurity concentration (i.e. ratio of impurity and main ion densities) strongly depends on the main ion flow pattern in the SOL, and is very sensitive to the details of this main ion flow pattern. The latter may depend on the divertor regime at the targets, NBI momentum torque, choice of anomalous transport coefficients, and so on. 

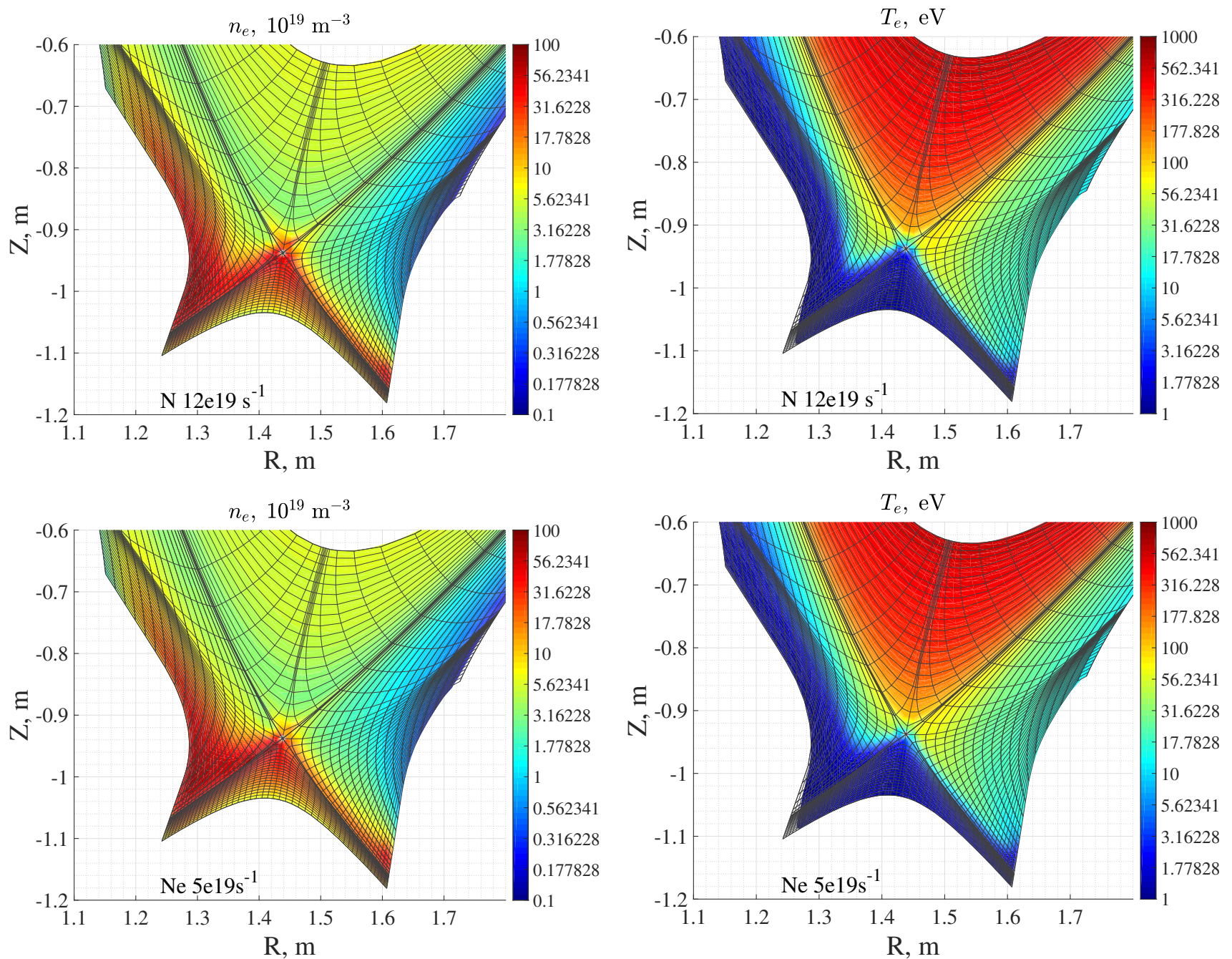

Figure 24. 2D contour plots of electron density and temperature in the vicinity of X-point and targets for cases with high seeding rate.

\section{Changes in impurity distribution with transition to detachment}

As the seeding rate increases and the outer target starts the transition to detachment, the impurity retention tends to be more pronounced. We illustrate this with the cases with nitrogen seeding rate of $1.2 \cdot 10^{20}$ atoms/s and neon seeding rate of $5 \cdot 10^{19}$ atoms/s. The corresponding $2 \mathrm{D}$ electron density and temperature profiles are plotted in Figure 24. One can see the significant temperature decrease near the strike point at the outer target, which may be used as the main indicator of the transition to detachment. Electron density increases correspondingly, becoming almost equal near the inner and outer targets. Note that this density rise near the outer target is accompanied by the reduction of the HFSHD region size near the inner target, i.e. main ions redistribution takes place. Also at the outer target the transition to detachment is not full.

A final remark is that similar plasma parameters (and also degree of detachment) may be reached with different nitrogen and neon seeding rates, and the neon seeding rate required is several times smaller than the nitrogen one.

The main ion source strength together with the direction of main ion poloidal flow is shown in Figure 25 for the nitrogen case with seeding rate $1.2 \cdot 10^{20}$ at $/ \mathrm{s}$ - for the corresponding neon case profiles are similar. Note that no stagnation point is seen at the HFS of the SOL - all ionized deuterium is returned back to the inner target. Simultaneously, neutrals born near the inner target 


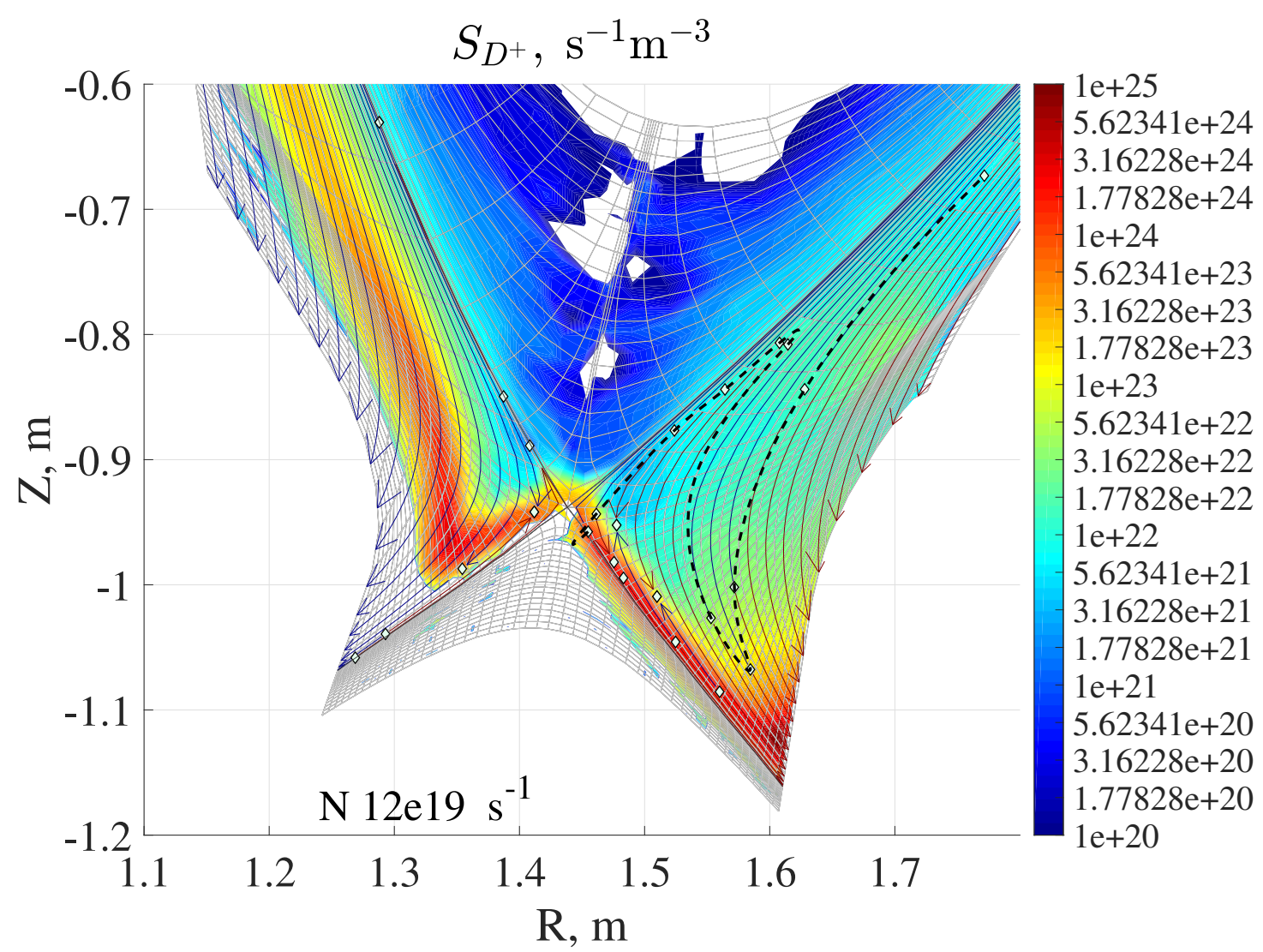

Figure 25. Deuterium ionization source strength from neutral to first ionization state (contour plot) and the direction of ion poloidal flow (red and blue arrows) for two cases with nitrogen and neon seeding. Meanings of colorbar and arrows are the same as in Fig. 8.

due to ion recombination, can reach the outer target through the PFR more easily, since i) ionization in the PFR is less pronounced; ii) even if ionization in the PFR takes place, the role of the $E \times B$ drift flow in returning ions back to the inner target becomes less significant.

As a consequence (see Figure 26), no upstream impurity flow forms near the inner target: the thermal force is not strong enough to shift the stagnation point towards to the target. Thus the leakage at the HFS becomes negligible.

At the LFS the retention becomes more pronounced with the increasing seeding rate, i.e. as the outer target transits to the detachment. This is caused by the increase of the electron density, which reduces the neutral ionization length and the probability for neutral atoms to penetrate above the stagnation point. Thus the fraction of neutrals which cannot reach the stagnation point increases — this may be seen in Figure 26 as a large ionization source strength below the stagnation point thus increasing the impurity retention.

As a measure of impurity compression we use the ratio of impurity density $n_{\mathrm{N}}$ or $n_{\mathrm{Ne}}$ (summed over all ionization states including neutrals) near the target to its midplane value at the separatrix. The resulting profiles along fixed flux tubes are shown in Figure 27. Note that the HFS density profiles are normalized to the HFS midplane density, while the LFS profiles are normalized to the LFS one. Thus one can see that the target/midplane density ratio indeed increases with the increasing seeding rate, while the target assymetry becomes less pronounced. Note that the plotted quantity differs from what is usually undestood as the compression (the ratio of neutral density in exhaust to the ion core density), but the advantage is that the divertor assymetry can be monitored, and that the poloidal inhomogeneity of the core impurity density does not affect the result. 

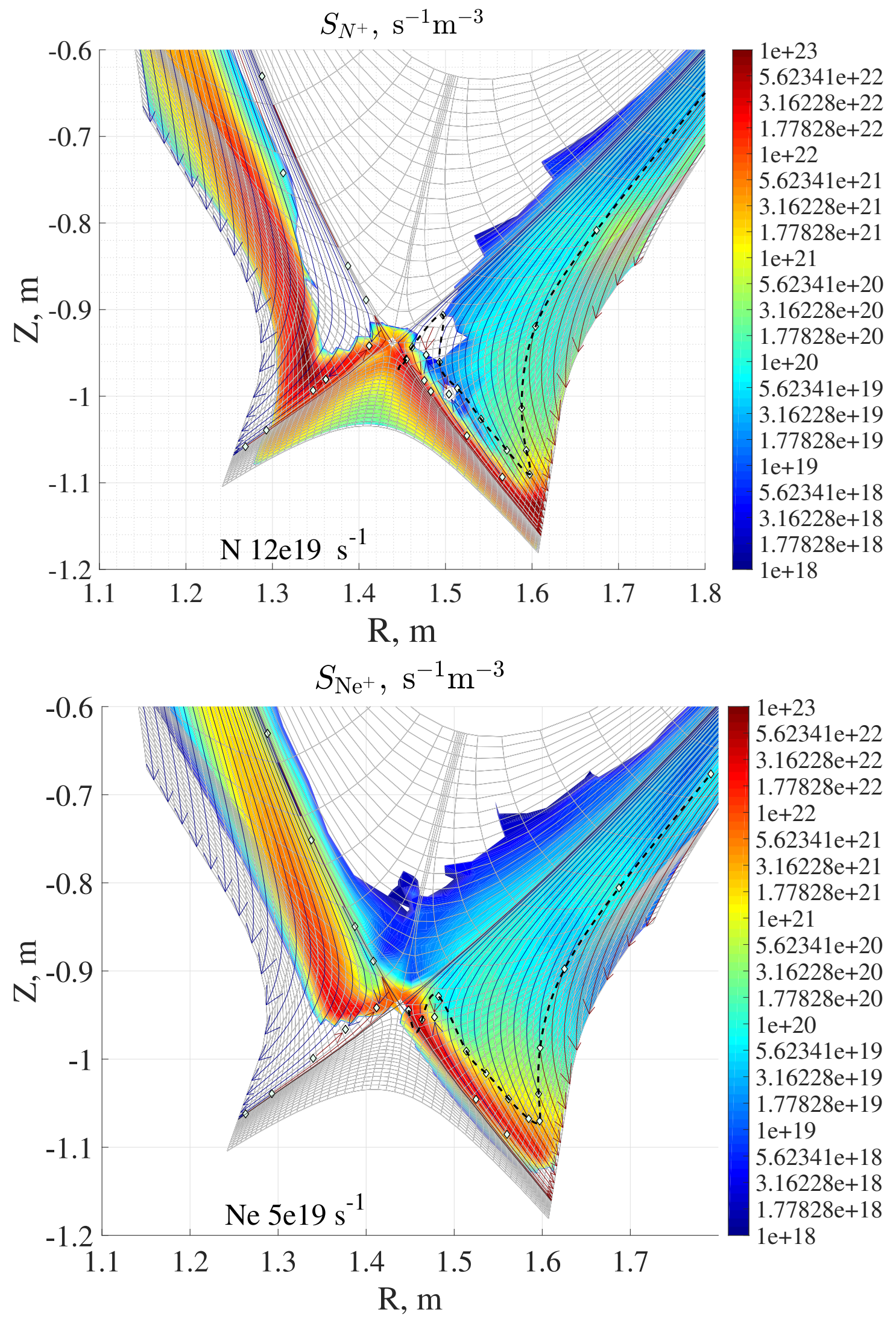

Figure 26. Impurity ionization source strength from neutral to first ionization state (contour plot)

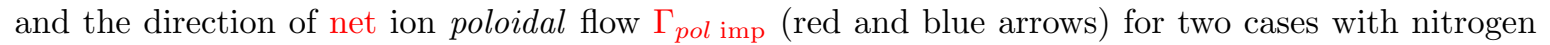
and neon seeding. Meanings of colorbar and arrows are the same as in Fig. 8. Mind that the colorbars differ from ones used in Fig. 10 due to bigger impurity density. 

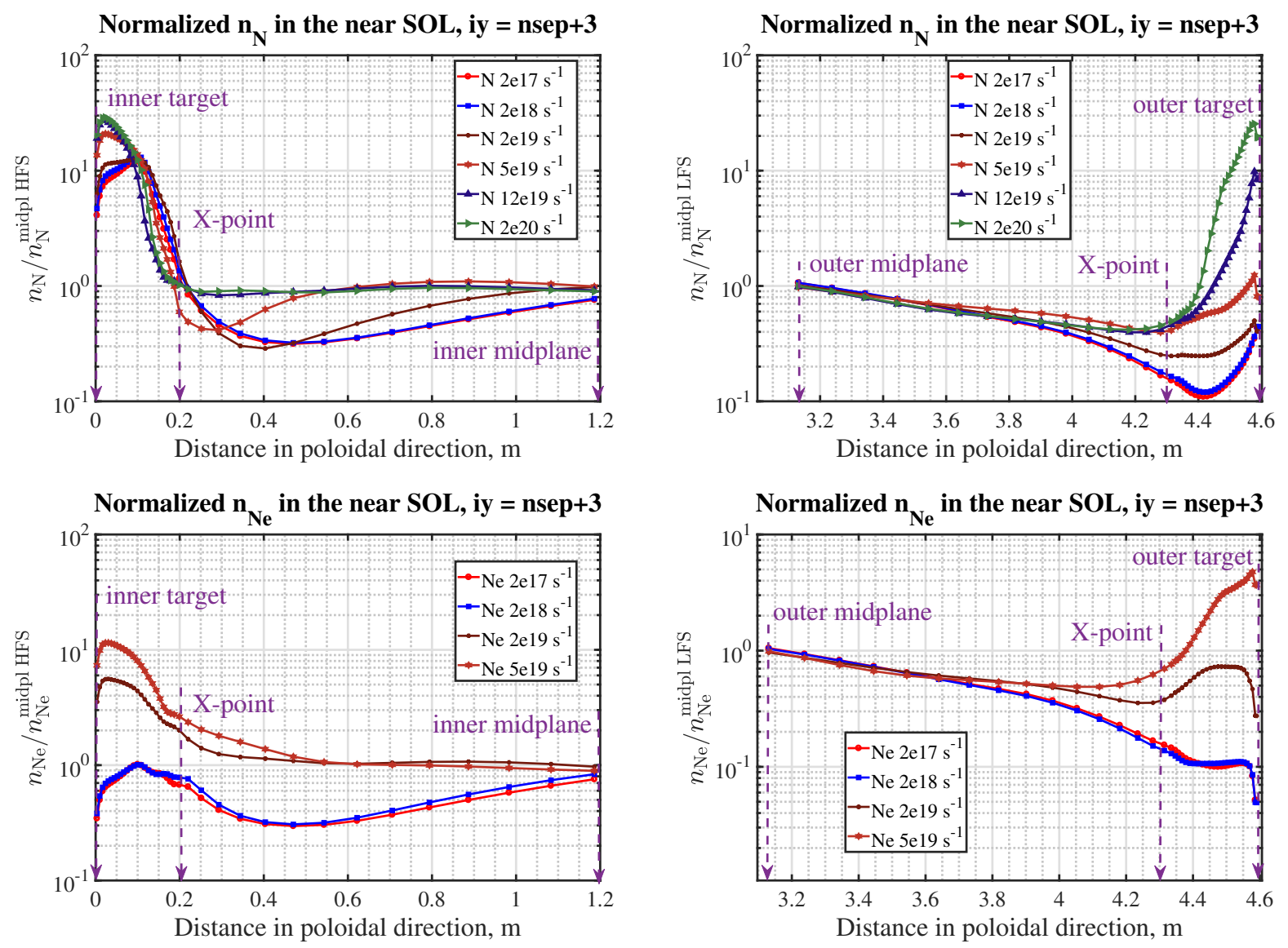

Figure 27. Ratio of impurity density $n_{\mathrm{imp}}$ to its value at the midplane $n_{\mathrm{imp}}^{\text {midpl }}$ plotted from target to midplane along the flux tube located at $0.87 \mathrm{~mm}$ from the separatrix (as measured at outer midplane). Poloidal distance is measured from the inner target. Note that the $n_{\mathrm{imp}}^{\mathrm{midpl}}$ are different at HFS and LFS.

\section{Conclusions}

Modeling of a typical ASDEX Upgrade discharge with nitrogen seeding is performed by means of the SOLPS-ITER 2D transport code with full account of drifts and currents. The attention is focused on the mechanisms of impurity leakage or retention. To study these mechanisms in more detail the corresponding modeling cases are run with the replacement of nitrogen by neon as a seeded radiator, keeping all other parameters of the simulation unchanged.

It is demonstrated that in most parts of the SOL and in almost all regimes the friction force of main ions balances the thermal force, and thus defines the difference between parallel velocities of the main ion and the impurity. However, the impurity leakage or retention is defined not by the competition of friction and thermal forces, but by the profiles of the impurity poloidal velocity and the impurity ionization source strength. The fraction of leaking impurity is equal to the fraction of neutrals which can reach the impurity poloidal velocity stagnation point before the ionization. Those neutrals which can penetrate above this stagnation point are dragged upstream, others are returned back to the target.

The impurity poloidal velocity is mainly defined by the poloidal projection of their parallel velocity and by the $E \times B$ drift velocity, where the former may be found from the impurity force balance. This force balance may be approximated with reasonable accuracy by the balance of friction force and thermal force, so that impurity parallel velocity depends on temperature gradients and main ion parallel velocity. Thus the distribution of the main ions flows, especially poloidal flows, determines 
the impurity distribution in the edge plasma. Since the former is to a large extent determined by the $\nabla B$ and $E \times B$ drifts, a correct physical pattern of the impurity flows can only be obtained in simulations including drifts. To prove this statement the corresponding runs are made with drifts switched off, and a drastic change in the main ion and the impurity distributions and flow patterns is noted.

Thus the main mechanism of impurity leakage is the coupling to the main ions due to friction, and impurity ion flux is directed upstream not only on those flux tubes where the main ion flux points upstream, but also in those regions where the thermal force is big enough to make the impurity flux directed upstream despite that the main ion flux being directed downstream.

In the modeled regimes of ASDEX Upgrade tokamak such an upstream main ion flux has a Pfisrch-Schlüter nature, it is closed through the core by $\nabla B$ drift flux. However, another mechanism of main ion upstream flux formation in the near SOL is so called reversal flows - they begin at the location of maximal ionization, then turn into radial diffusive fluxes at or near the midplane, and finally return back to the target along the B-field in the far SOL. Note that the predominance of Pfisrch-Schlüter flow over the reversal flow is not a general result, this might not be the case for another regimes and another machines.

The leakage efficiency of impurities depends on their ionization potential, or, more precisely, on their ionization rate $\left\langle\sigma_{\text {ion }} v\right\rangle$, since it defines the ionization source strength. Those impurities which have the bigger ionization potential, have a larger probability to penetrate above their poloidal velocity stagnation point before the ionization, from where they are dragged upstream.

The general prediction is that the leakage efficiency is higher for neon than for nitrogen and deuterium, because the neon ionization source maximum is located further upstream due to its higher ionization potential, while the neon poloidal stagnation point is shifted closer to the target with respect to the deuterium one due to thermal force. For nitrogen (as compared to deuterium) two these effects act in opposite directions, but the effect of stagnation point shift is found to be bigger for modeled regimes thus making the nitrogen leakage efficiency bigger than the deuterium one. As a result, the neon upstream density is expected to be higher than the nitrogen one, and this is supported by simulations.

This qualitatively agrees with the experimental results obtained on many machines that the nitrogen is compressed near the targets providing high radiative losses there without significant core confinement deterioration. Neon, in contrast, tends to penetrate upstream, which makes it difficult to find an operational window with simultaneous radiative divertor operation and high core confinement with neon seeding. Simulations also demonstrate that, to obtain similar profiles at the targets and a similar degree of detachment, a smaller neon seeding rate is required than for nitrogen, typically by 2.5 times.

\section{Acknowledgments}

The work was done at the Peter the Great Saint Petersburg Polytechnic University under support of the Russian Scientific Foundation, Grant No. 17-12-01020. Numerical calculations were performed at the Polytechnic SuperComputer Center at Peter the Great St. Petersburg Polytechnic University. Authors thank Dr Richard Pitts for his fruitful comments and suggestions during the work on the present paper. The views and opinions expressed herein do not necessarily reflect those of the ITER Organization. 


\section{References}

[1] P. Stangeby, "The plasma boundary of magnetic fusion devices" 2000 IOP publishing Ltd, pages 296-323.

[2] D.-K. Kim and S. H. Hong 2005 Physics of Plasmas 12, 062504

[3] R.A. Pitts et al 2011 Journal of Nuclear Materials 415 S957-S964

[4] A.R. Raffray et al 2010 Fusion Eng. Des. 85 93-108

[5] S. Potzel et al 2014 Nucl. Fusion 54013001

[6] F. Reimold et al 2015 Nucl. Fusion 55033004

[7] A. Kallenbach et al 2015 Nucl. Fusion 55053026

[8] A.R. Field et al 2017 Plasma Phys. Control. Fusion 59095003

[9] C Guillemaut et al 2017 Plasma Phys. Control. Fusion 59045001

[10] M.L. Reinke et al 2011 Journal of Nuclear Materials 415 S340-S344

[11] M. Bernert et al 2017 Nuclear Materials and Energy 12 111-118

[12] J. A. Goetz et al 1999 Physics of Plasmas 61899

[13] T. W. Petrie et al 2007 Journal of Nuclear Materials 363-365 416-420

[14] X. J. Liu et al 2017 Physics of Plasmas 24122509

[15] G. Telesca et al 2017 Nucl. Fusion 57126021

[16] F. Reimold et al 2015 Journal of Nuclear Materials 463 128-134

[17] F. Reimold et al 2017 Nuclear Materials and Energy 12 193-199

[18] F. Reimold et al 2015 ITPA meeting....

[19] R. Schneider et al 2006 Contrib. Plasma Phys. 46 3-191

[20] A. S. Kukushkin et al 2011 Fusion Engineering and Design 86 2865-2873

[21] S. Wiesen et al 2015 Journal of Nuclear Materials 463 480-484

[22] X. Bonnin et al 2016 Plasma and Fusion Research 111403102

[23] V. Rozhansky et al 2009 Nucl. Fusion 490250007

[24] D. Reiter. The EIRENE Code User Manual. Available at http://www.eirene.de

[25] E. Sytova et al 2018 Contrib. Plasma Phys. https://doi.org/10.1002/ctpp.201700135.

[26] S. Krasheninnikov and A. Kukushkin 2017 J. Plasma Phys. 83, 155830501

[27] V. Rozhansky 2014 Contrib. Plasma Phys. 54 508-516

[28] S. I. Braginskii, in Reviews in Plasma Physics, edited by M. A. Leontovich (Consultants Bureau, New York, 1965), Vol. 1, p. 205.

[29] V. Zhdanov, Transport Processes in Multicomponent Plasma, RC Press, Boca Raton, FL 2002 ISBN-10 0415279208.

[30] S.K. Erents et al 2004 Plasma and Fusion Research 461757

[31] H.W. Müller et al 2005 Proc. 32 $2^{\text {nd }}$ nd EPS Conf. on Plasma Phys. and Controlled Fusion, Tarragona 2005 ECA Vol. 29C P1.009

[32] H.W. Müller et al 2007 Journal of Nuclear Materials 363-365 664

[33] B. Labombard 2009 Nucl. Fusion 441047

[34] N. Asakura et al 2004 Nucl. Fusion 44503

[35] R.A. Pitts et al 2007 Journal of Nuclear Materials 363-365 575

[36] N. Asakura et al 2007 Journal of Nuclear Materials 363-365 41

[37] P. Molchanov et al 2008 Plasma and Fusion Research 50115010

[38] H.P. Summers et al 2002 Plasma and Fusion Research 44 B323

[39] V. Rozhansky et al 2018 Contrib. Plasma Phys. https://doi.org/10.1002/ctpp.201700119

[40] A.E. Jaervinen et al 2017 Nuclear Materials and Energy 12 1136-1140

[41] L. Aho-Mantila et al 2017 Plasma Phys. Control. Fusion 59035003

[42] G.S. Kirnev et al Journal of Nuclear Materials 337-339 271

[43] L. D. Horton et al 2005 Nucl. Fusion 44856

[44] D. Galassi et al 2017 Nucl. Fusion 57036029 